\title{
Adaptation and coordinated evolution of plant hydraulic traits.
}

\author{
Pablo Sanchez-Martinez ${ }^{1}$, Jordi Martinez-Vilalta ${ }^{1}$, Kyle Dexter ${ }^{2}$, Ricardo Segovia ${ }^{2}$, and \\ Maurizio Mencuccini ${ }^{3}$ \\ ${ }^{1} \mathrm{CREAF}$ \\ ${ }^{2}$ University of Edinburgh Department of Geology and of Geophysics \\ ${ }^{3}$ Institucio Catalana de Recerca i Estudis Avancats
}

May 5, 2020

\begin{abstract}
Hydraulic properties control plant responses to climate and are likely to be under strong selective pressure, but their macroevolutionary history remains poorly characterized. We compiled a global dataset of hydraulic traits describing xylem efficiency, xylem safety, sapwood allocation relative to leaf area and drought exposure and matched it with a newly derived genus-level phylogeny to shed light on woody-plant hydraulic eco-evolutionary patterns. All hydraulic traits present medium to high levels of phylogenetic signal, being evolutionarily segregated into two phylogenetically conserved adaptive modules: the safety-exposure coordination, whereby lineages exposed to drought adapted to withstand low water potentials by evolving a xylem with higher embolism resistance; and the efficiency-allocation coordination, whereby higher water availability and deeper, water-retentive soils led to the evolution of hydraulically efficient species with higher leaf area relative to sapwood area. Moreover, the lack of evolutionary correlation between xylem safety and efficiency suggest that both adaptive modules are independent.
\end{abstract}

\section{Introduction}

Water transport in plants occurs under negative pressure (tension) and is driven by the process of transpiration at the leaf-atmosphere interface, which generates a water potential depression throughout the plant (cohesion-tension theory) (Dixon 1914; Tyree \& Zimmermann 2002). A key source of vulnerability for the water transport system is the formation of xylem embolism, resulting from the breakage of the water columns caused by cavitation (the phase change from liquid water to gas), which reduces hydraulic conductivity and may lead to plant death through hydraulic failure (Tyree \& Zimmermann 2002). This process is more likely to occur during drought events, as low water availability jointly with high evaporative demand results in low plant water potentials. In fact, a wealth of research over the last decades has established that hydraulic failure is a principal mechanism triggering tree mortality under drought (Adams et al. 2017; Choat et al. 2018). Therefore drought, together with other important sources of selection such as freezing temperatures (Zanne et al. 2014), has been considered one of the primary forces shaping plant evolution by acting directly on hydraulic traits (Maherali et al. 2004; Larteret al. 2017; Zheng et al. 2019).

Vulnerability to xylem embolism is normally quantified by determining the water potential that causes $50 \%$ reduction in hydraulic conductivity (P50) (Choat et al. 2012, 2018). Because species differ greatly in their exposure to low water potentials, the actual hydraulic risk is normally represented by the hydraulic safety margin (HSM), which is the difference between the minimum water potential experienced $\left(\psi_{\min }\right)$ and P50. $\psi_{\min }$ emerges from the balance between soil water availability, the rate of leaf water loss, and the capacity of plant transport system to supply water to leaves, and it is thus determined by plant functional aspects such as rooting strategies and stomatal control as well as by abiotic factors (Bhaskar \& Ackerly 2006). P50 and $\psi_{\text {min }}$ are known to co-vary, leading to relatively invariant HSMs at the global scale (Choat et al. 2012). Since hydraulic risk is likely to be under greater selective pressure than $\psi_{\min }$ and P50 per se, these two 
latter traits are expected to be coordinated, or correlated, over the evolutionary history of lineages, being shaped by similar selective pressures.

Transport efficiency is another key determinant of hydraulic performance. Xylem efficiency in water transport is usually quantified as the maximum, stem-specific hydraulic conductivity $\left(K_{\mathrm{s}}\right)$. Because the structural properties of conduits and pit membranes associated with increased embolism resistance (quantified here as P50) also tend to reduce conductive efficiency, a trade-off between P50 and $K{ }_{\mathrm{s}}$ has long been hypothesized (Tyree \& Zimmermann 2002). Although this trade-off has been shown to be relatively weak at the species level (e.g. Maheraliet al. 2004; Jacobsen et al. 2007; Gleason et al.2016), it has seldom been analysed accounting for phylogenetic relatedness of taxa at the global scale. It seems reasonable to hypothesize that evolutionary processes associated with frequent drought occurrence would have driven an increase of xylem safety, allowing taxa to bear lower water potentials and ensuring hydraulic performance at the expense of efficiency (Jacobsen et al. 2007). In contrast, increased xylem efficiency could have evolved in environments where a higher conductivity was adaptive, and selective pressures favouring expensive safety features were weaker (Tyree \& Zimmermann 2002).

The role of hydraulic efficiency is more nuanced when considered at the whole plant level, where transport capacity needs to match water demand, which is in turn strongly influenced by leaf area (Mencuccini et al. 2019). Consequently, xylem conductive capacity is frequently expressed in a relativized manner as a measure of hydraulic sufficiency (leaf-specific hydraulic conductivity; $K_{1}$, where $K_{1}=K_{\mathrm{s}} * \mathrm{Hv}_{\text {) }}$ (Tyree \& Zimmermann 2002). From this perspective, plants may adapt to drought stress prioritizing supply over demand by increasing the ratio of cross-sectional sapwood area relative to leaf area (i.e., their Huber value; Hv) (Mencuccini et al. 2019). Contrarily, lineages not exposed to drought stress and with no restriction to evolve towards a more efficient xylem may be able to supply water to a higher leaf area by using a relatively low sapwood area, potentially allowing for higher productivity (Maherali et al. 2004; Choat et al. 2012; Larter et al. 2017).Therefore, we would also expect xylem efficiency and sapwood-to-leaf allocation to be coordinated over evolutionary timescales, responding to the drought stress experienced by lineages and maintaining relatively constant values of $K_{1}$ across diverse environmental conditions.

We thus hypothesize that these three pairs of hydraulic traits (i.e., P50- $\psi_{\text {min }}, \mathrm{P} 50-K_{\mathrm{s}}, K_{\mathrm{s}}-\mathrm{Hv}$ ) represent adaptive modules, specifically meaning that they show correlated evolution. Moreover, each of these traits is hypothesized to respond to environmental selective pressures by means of adaptation (Ackerly et al. 2000; Reich 2014). At the same time, as these traits show a wide range of values and our study species are spread broadly across woody plant lineages and environmental conditions, we also expect to find evidence for evolutionary constraints. This would be reflected in significant phylogenetic signal, whereby closely related species will have more similar trait values than expected by chance (Losos 2008) (see Fig. S1 in Supporting Information for hypotheses graphical description). To test these hypotheses, we first describe variation in woody-plant hydraulic traits across species, disentangling to what extent it is related to phylogeny. We then examine the relationships between traits and environmental conditions. Next, to assess coordinated evolution between traits and adaptation to selective pressures, we quantify the evolutionary correlations among hydraulic traits and between hydraulic traits and environmental drivers. Finally, phylogenetic mapping of the traits involved in adaptive modules is employed to qualitatively display patterns of evolutionary coordination.

\section{Materials and methods}

\section{Data sources}

We extracted detailed hydraulic trait data from a database covering 2172 species from 833 genera. Most of the data comes from a previously published database (Mencuccini et al. 2019), which was supplemented with the database reported by Liu et al . (2019). To enforce a common taxonomy across datasets, names were matched against accepted names in The Plant List using the "taxonstand" package (Cayuela et al. 2012). Then, the "taxonlookup" package (Pennellet al. 2016) was used to complete species information at the genus, family, order and major evolutionary affiliation (Angiosperms vs. Gymnosperms) levels. The database covers 
all major biomes (Fig. S2, Fig S3), and after filtering out non-woody plants and Pteridophytes, 2105 species were retained for subsequent analyses (1957 Angiosperms and 148 Gymnosperms).

We used data of four hydraulic traits that were represented across a sufficiently large numbers of species (N > 550): (1) stem-specific hydraulic conductivity $\left(K_{\mathrm{s}}, \mathrm{kg} \mathrm{m}^{-1} \mathrm{MPa}^{-1} \mathrm{~s}^{-1}\right)$ as a measure of xylem efficiency; (2) water potential at $50 \%$ loss of hydraulic conductivity (P50, MPa) as a measure of xylem safety; (3) twigbased Huber value $\left(\mathrm{Hv} ; \mathrm{cm}^{2} \mathrm{~m}^{-2}\right)$, defined as the sapwood cross-sectional area to leaf area ratio, as a measure of allocation; (4) minimum midday leaf water potential $\left(\psi_{\min }, \mathrm{MPa}\right)$ as a measure of exposure to drought stress. We also include variables integrating two pairs of the four selected traits, specifically, (5) leaf-specific hydraulic conductivity $\left(K_{1}, \mathrm{~kg} \mathrm{~m}^{-1} \mathrm{MPa}^{-1} \mathrm{~s}^{-1}\right)$ as the hydraulic capacity per unit leaf area (the product of $K$ $\mathrm{s}$ and $\mathrm{Hv}$ ) and (6) the hydraulic safety margin (HSM) as the difference between $\psi_{\min }$ and P50 (Table S1). When multiple measures for one species were available, mean values were used for all traits except for $\psi_{\min }$, where the minimum was used. For all variables, we excluded data from seedlings and studies in greenhouses or experimental gardens. Data obtained on roots and leaves were also excluded (Liu et al. 2019; Mencuccini et al. 2019).

A total of 16 environmental variables were compiled (10 related to climate and six to soil properties) (Table S1). All climatic variables were extracted from WorldClim (Fick \& Hijmans 2017) (www.worldclim.org; accessed on February 2019) except for Moisture Index, which was extracted from the global aridity and potential evapotranspiration (PET) database (Trabucco \& Zomer 2018) (http://www.cgiar-csi.org, data accessed on February 2019) at a resolution of $30 \operatorname{arcsec}(\sim 1 \mathrm{~km}$ at the equator). Soil data were extracted from SoilGrids (Ribeiro et al. 2017) (https://soilgrids.org/, accessed on February 2019) at the same resolution. Occurrences for all species were obtained from the Global Biodiversity Information Facility (GBIF) and the Atlas of Living Australia (ALA) using the "speciesmap" package (https://remkoduursma.github.io/speciesmap/articles/Using_speciesmap.html).

\section{Phylogeny}

We used a genus-level phylogeny instead of a species-level one to avoid issues with species misidentifications, which are particularly common in the tropics (Goodwin et al. 2015; Baker et al. 2017), where a considerable amount of our hydraulics data comes from (Fig. S2 and S3). Some models, however, were also fitted using the species-level phylogeny from Smith \& Brown (2018) to assess the robustness of our results to the taxonomic resolution of our phylogenetic data (see below). To construct the genus-level phylogeny, sequences of the $r b c \mathrm{~L}$ and mat K plastid gene for 707 Angiosperm tree genera were obtained from Genbank (www.ncbi.nlm.nih.gov/genbank/) building on previous efforts (Dexter \& Chave 2016; Neves et al. 2020; Segovia et al. 2020). Sequences were aligned using the MAFFT software (Katoh \& Standley 2013). "Ragged ends" of sequences that were missing data for most genera were manually deleted from the alignment. The two chloroplast markers were concatenated, and a maximum likelihood phylogeny for the genera was estimated in the RAxML v8.0.0 software (Stamatakis et al. 2008), on the CIPRES web server (www.phylo.org), using General Time Reversible (GTR) + categorical Gamma (G) model of sequence evolution. The tree was constrained following the order-level phylogeny, based on the topology proposed by the Angiosperm Phylogeny Group IV (Gastauer \& Meira Neto 2017). Sequences of Nymphaea alba (Nymphaeaceae) were included as outgroup.

The resulting maximum likelihood phylogeny for Angiosperms was temporally calibrated using the software treePL (Smith \& O'Meara 2012). Age constraints for internal nodes were implemented for most families and orders (Magallón et al. 2015). The rate smoothing parameter (lambda) was set to 10 based on a cross-validation procedure. Finally, the newly-derived Angiosperms phylogeny was fused with an existing Gymnosperm phylogeny (Leslie et al. 2018). We manually added the genera Gnetum and Ginkgo according to ages found in the literature, $174 \mathrm{Ma}$ for the Gnetales (Ran et al. 2018) and 265.2 Ma for Ginkgoaceae (Tank et al. 2015).

\section{Statistical analyses}

All analyses were carried out in R (3.6.0) (R Core team 2019). Some variables were transformed to achie- 
ve normality (absolute values in the case of P50 and $\psi_{\min }$ ) (Table S1). As many environmental variables were highly correlated with one another, a Principal Components Analysis (PCA) on the 16 variables was performed using the R package "stats" (R Core team 2019) to reduce the number of axes summarizing environmental variation. The first principal component explained $41.7 \%$ of the variance, representing variation in water availability and some related variables such as soil $\mathrm{pH}$, with high values characterizing more humid locations with leached acidic soils (Table S2, Fig. S4). The second principal component explained $23.3 \%$ of the variance, representing variation in energy input, with high values characterizing low solar irradiation and low atmospheric water demand (Tables S2, Fig. S4). Finally, the third principal component accounted for $13.6 \%$ of the variance and was mainly represented by soil properties, with high values indicating shallower, sandier soils, and by maximum wind velocity (positive correlation with axis) (Table S2, Fig. S4). The remaining components explained a low proportion of variance $(<6 \%)$, so the first three axes were used to characterize the environmental niches of species in the following analyses.

Univariate and bivariate phylogenetic mixed models, alternatively including or excluding fixed effects of environmental principal components, major evolutionary affiliation (Angiosperm vs. Gymnosperm) and their interactions were fitted using the "MCMCglmm" R package (Hadfield 2010) (see Table S3 for models description). All models accounted for the occurrence of multiple measurements in each terminal taxon (in this case, multiple species measures for each genus) by the inclusion of genus identity as a random effect. Moreover, genus-level phylogenetic relationships were taken into account as a second random effect using the previously presented phylogeny. The inclusion of these random effects allowed us to partition of the residual variance from models into three components: the inter-generic variance due to phylogenetic relationships; the non-phylogenetic, inter-generic variance; and the intra-generic variance. The inter-generic phylogenetic variance quantifies the variability explained by the relationships among taxa as given by our phylogenetic hypothesis and, when divided by the total variance, gives a measure of the phylogenetic signal $(\lambda)$ (Lynch 1991). Non-phylogenetic inter-generic variance $(\gamma)$ accounts for the proportion of among-genus variability not explained by the phylogeny, and the intra-generic variance $(\rho)$ provides a measure of the proportion of variability due to intra-generic trait variation (plus any residual error) (Hadfield \& Nakagawa 2010) (see Appendix S1 for a more formal description). Specifically, $\rho$ accounts for what we call "late burst" diversification, which gives a measure of the proportion of the variability that is caused by more recent trait changes among species within genus, which could be related to phylogenetic relationships within genera or could represent non-phylogenetically related variance among species.

To partition variances of phylogenetic and non-phylogenetic components, we implemented univariate models without fixed effects for the six selected hydraulic traits and for the three environmental PCA axes (Table 1). To identify relationships between hydraulic traits and environmental PCA axes, we then ran univariate models with hydraulic traits as response variables and single environmental principal components as fixed effects, both accounting and not accounting for phylogenetic relationships affecting the response trait. To examine the effect of the major split between Angiosperms and Gymnosperms, some of these models included a binary variable describing major evolutionary affiliation and the interaction between affiliation and environment. For each group of nested models, the best fitting one in terms of DIC (Deviance Information Criterion) was selected (Fig. 1, Table S6 to see DIC values). Models within 4 DIC units of each other were considered equivalent in terms of fit and the simplest one was selected.

Subsequently, to characterize phylogenetic covariation between the hydraulic variables involved in the three hypothesized evolutionary correlations $\left(\mathrm{P} 50-\psi_{\min }, \mathrm{P} 50-K_{\mathrm{s}}\right.$ and $\left.K_{\mathrm{s}} \mathrm{Hv}\right)$ and between each hydraulic variable and the three environmental principal components, bivariate models were used. In these models, two response variables and their phylogenetic structure were considered simultaneously, resulting in a variancecovariance matrix from which the evolutionary correlation between the two variables could be calculated (Appendix S1). Evolutionary correlations were calculated between pairs of traits, including and excluding the three environmental components, evolutionary affiliation and their interactions as fixed effects (Table 2, Fig. S2 and Fig. S3 to see data coverage for each combination of traits). Finally, we also estimated evolutionary correlations between traits and single environmental principal components (Fig. 2, Table S5 to see correlations without fixed effects and Table S7 to see all correlations). All bivariate models were 
also implemented using the species-level phylogeny reported by Smith \& Brown (2018) and available in the R package "v.PhyloMaker" (Jin \& Qian 2019) covering the same observations (Appendix S2) to test for consistency with genus-level results.

"MCMCglmm" implements a Bayesian approach, estimating the posterior distribution of parameters, from which $95 \%$ credible intervals can be obtained (Hadfield 2010). We set independent normal prior distributions for fixed effects and non-informative Inverse-Gamma prior distribution for random effects and residual variances (Villemereuil \& Nakagawa 2014). Less informative expanded priors were also used, and highly similar results were obtained. Models were specified to achieve convergence while minimizing correlation between iterations (Appendix S1). Marginal variance explained $\left(\mathrm{R}_{\mathrm{m}}^{2}\right.$, variance explained by the fixed effects) and conditional variance explained $\left(\mathrm{R}_{\mathrm{c}}^{2}\right.$, variance explained by both fixed and random effects), were calculated for the single response models (Nakagawa \& Schielzeth 2013).

Finally, reconstructions of trait evolution under a Brownian motion model were mapped along the phylogeny using maximum likelihood ancestral state reconstructions (Schluter et al. 1997) by means of the "Phytools" R package (Revell 2013) (Fig. 3 and Fig. 4). Phylogenetic reconstructions for the three main environmental principal components and for HSM and $K_{1}$ were also conducted (Fig. S5 and Fig. S6, respectively).

\section{Results}

\section{Phylogenetic and non-phylogenetic variances}

All six selected traits showed a significant phylogenetic signal. The proportion of variance that was explained by the inter-generic phylogenetic structure $(\lambda)$ ranged from $0.432\left(K_{1}\right)$ to $0.745\left(\psi_{\text {min }}\right)$ (Table 1 and Fig. 2 ). This means that $43.2-74.5 \%$ of trait variances can be largely attributed to relatively deep evolutionary differences among genera, with the rest being attributed to non-phylogenetically correlated inter-generic $(\gamma)$ and intra-generic $(\rho)$ variances (Table 1 and Fig. 2$)$. Intra-generic variances $(\rho)$ ranged from $0.189\left(\psi_{\min }\right)$ to $0.532\left(K_{1}\right)$, being the second most important variance component in all cases, indicating that "late-burst" trait diversification within genera is a substantial generator of global trait variability. Meanwhile, species level analyses showed that variation within genera also show strong phylogenetic patterns (Appendix S2). Intergeneric, non-phylogenetically related variances $(\gamma)$ ranged from $0.036\left(K_{1}\right)$ to 0.225 (P50) and accounted for the lowest proportion of the variance in all cases (Table 1, Fig. 2 and Table S4 for non-phylogenetic models variance partition). Phylogenetic mapping of hydraulic traits qualitatively confirmed the findings reported above, showing more gradual changes in highly conserved traits such as $\psi_{\min }$ and changes more concentrated at the tips of the phylogeny for variables showing a lower phylogenetic signal, such as $\mathrm{Hv}$, which also showed higher late-burst diversification (Fig. 3 and Fig. 4).

Importantly, the phylogenetic signal of the three environmental principal components was also very high, particularly for PC1 (0.820) and PC3 (0.841) (Table 1, Fig. S5). These highly conserved principal components explained part of the apparent phylogenetic variance of hydraulic traits when considered as fixed effects, and some of them were evolutionarily correlated with hydraulic traits, as we report below (Table 2 and Fig. 2).

\section{Environmental drivers of hydraulic trait variability}

In models that accounted for phylogenetic structure, all hydraulic traits showed significant relationships with at least one of the three environmental axes defined by the PCA (Fig. 1). Conditional explained variances $\left(R^{2}{ }_{c}\right)$ were notably higher than marginal explained variances $\left(R^{2}{ }_{m}\right)$, indicating that accounting for the phylogenetic relationships was crucial to improve model fit (Fig. 1, see Table S6 for phylogenetic and non-phylogenetic explained variances).

Hydraulic safety (P50) was only related to PC1, negatively so, showing that species inhabiting drier habitats tended to be more resistant to embolism (Fig. 1). Minimum water potential at midday $\left(\psi_{\min }\right)$ was negatively related to all three environmental axes, confirming the pattern that species living in drier, more waterdemanding and windier habitats with shallower, sandier soils tended to be more exposed to drought stress (Fig. 1). However, only Angiosperms showed a significant relationship of $\psi_{\min }$ with PC1. $K_{\mathrm{s}}$ was found 
to be positively related to PC1 and negatively related to PC3, with the opposite being the case for Hv, implying that species inhabiting more humid areas with deeper soils and lower wind velocity were able to allocate more carbon to leaves relative to xylem while also having a more efficient xylem (Fig. 1). In addition, $K_{s}$ was marginally related to PC2. The hydraulic safety margin (HSM) was positively related to the three environmental components, with species inhabiting more humid environments with a lower energy input, shallower soils and higher wind velocities having a higher safety margin (Fig. 1). Nevertheless, the relationships between HSM and PC1 and PC2 were negative for Gymnosperms, suggesting that for this clade, the higher the water availability and the lower the energy input, the lower the safety margin. Leafspecific conductivity $\left(K_{1}\right)$ was only related to PC2, reaching higher values with a more water-demanding atmosphere, and marginally negatively related to PC1 in the case of Gymnosperms (Fig. 1).

\section{Evolutionary correlations}

Two of the six possible evolutionary correlations between pairs of traits were significant, coinciding with two of the three hypothesized coordinations. These were the ones involving (1) P50 and $\psi_{\text {min }}$ (positive correlation) and (2) $K_{s}$ and $\mathrm{Hv}$ (negative correlation) (Fig. 2, Table 2). A significant positive evolutionary correlation between $\mathrm{Hv}$ and P50 was found, but its significance disappeared once environmental principal components were accounted for as fixed effects. A significant evolutionary correlation between P50 and $K_{s}$ was also obtained when using the species-level phylogeny and also disappeared when including environmental principal components as fixed effects. Thus, these apparent correlations ( $\mathrm{Hv}-\mathrm{P} 50$ and $\left.K_{s}-\mathrm{P} 50\right)$ are likely explained by the similarity in the response of both traits to environmental conditions (Table 2, Appendix S2). No evolutionary correlation was found between any other pair of traits (Fig. 2, Table S7).

Consistent evolutionary correlations were also observed between certain hydraulic traits and environmental principal components in the bivariate models: $K$ s was positively correlated with PC1 (water availability), and negatively with PC3 (soil properties); Hv was negatively correlated with PC1 and positively with PC3; and both $|\mathrm{P} 50|$ and $\left|\psi_{\text {min }}\right|$ were negatively correlated with PC1 (Fig. 2, Table S5). When using the species level phylogeny, a marginally significant relationship between $K_{\mathrm{s}}$ and PC2 was also reported (Appendix S2). These results were similar but not identical to those reported by the univariate-response models shown in Figure 1. Specifically, the relationships between $\psi_{\min }$ and PC2 and PC3 reported in univariate models were not supported as evolutionary correlations.

\section{Discussion}

\section{Conservatism in hydraulic trait evolution}

We found a clear pattern of phylogenetic conservatism for hydraulic traits in our study, suggesting that the legacy of traits found in species' evolutionary ancestors is an important determinant of trait values in extant species. While we cannot formally rule out Brownian motion evolution operating over long evolutionary timescales as the source of present-day trait variability on the basis of our single trait variance partitioning (Losos 2008; Revell et al. 2008), our finding of evolutionary correlations of traits with environmental variables illustrates a key role of non-random evolutionary processes. Moreover, environmental components explained part of traits' phylogenetic variance when accounted for as fixed effects (Table 1 and Table 2). Our analyses indicate that adaptive processes have driven the diversification of hydraulic traits, while the prevalent pattern of phylogenetic niche conservatism suggest that evolutionary constraint has limited the range of trait values within lineages (Crisp \& Cook 2012). If no evolutionary constraint were operating in plant lineages and exposure to novel environmental regimes occurred, lineages would shift their hydraulic traits in response to new selective pressures, and there would be little phylogenetic signal for traits and environmental conditions in modern species, i.e. we would not find a pattern of phylogenetic niche conservatism. As dispersal and exposure to novel environmental conditions are not limited at the evolutionary timescales considered here (Crisp et al. 2009; Segovia et al . 2020), the phylogenetic signal reported likely results from evolutionary constraints operating within lineages via biophysical limitations (e.g., related to the role of xylem in the mechanical support of woody plants (Niklas 2007)), absence of genetic and phenotypic variation upon which selection can act (Lamy et al. 2014) antagonistic genetic variation (Etterson \& Shaw 2001), or other processes 
(e.g., pleiotropy).

The strong and significant evolutionary correlations that were found between environmental variables and hydraulic traits (Fig. 2 and Table 2) suggests that traits have changed in a predictable manner as lineages shifted across environments over evolutionary time. This pattern is congruent with the conservatism of niches across lineages at the study scale, not only based on the observed trait phylogenetic signal, but on the conservatism of environmental conditions and evolutionary correlations between environmental niche axes and the studied functional traits. Thus, species may have been tracking environments similar to those their ancestors were already adapted to, retaining ancestral traits due to stabilizing selection (Willson et al. 2008; Ackerly 2009). Nevertheless, we do observe a wide range of trait values across lineages (including genera), indicating that adaptive divergence of lineages has occurred in deep evolutionary time (Fig. 3, Fig. 4, Fig. S5 and Fig. S6). Further, substantial trait variation can also arise over shorter evolutionary timescales (e.g., in recent evolutionary time) via species adapting to present-day selective pressures, as reported by the significant degree of variance at the intra-genus level (Table 1).

It is worth noting that our global eco-evolutionary overview may be limited by the availability of hydraulic data. Different evolutionary processes may be dominant depending on the taxon studied. For instance, hydraulic safety has been reported to be extremely labile for the genus Callitris (Larter et al. 2017) and to be conserved forJuniperus (Willson et al. 2008), while showing a high canalization for Pinus species (Lamy et al. 2014). Also, it is worth noting that phylogenetic mixed models are underlain by a Brownian motion model of evolution, which may not accurately describe the evolutionary processes shaping traits (Housworth et al.2004). Other evolutionary models, such as the Ornstein-Uhlenbeck model for selection towards optimal trait values (Butler \& King 2004), should be explored in future works.

\section{Evolutionary coordination and adaptation in plant hydraulics}

We report two clear evolutionary correlations between hydraulic traits, which remain significant once environment and major evolutionary affiliation of species are accounted for as fixed effects. Both of the pairs of evolutionarily correlated traits are likely to be functionally, developmentally and/or genetically integrated, as our analyses reject the possibility that the reported evolutionary correlations are a mere artefact of similar responses to environmental conditions or are caused by fundamental differences between Gymnosperms and Angiosperms. The first correlation involves hydraulic safety and the minimum water potential experienced by species (P50- $\left.\psi_{\min }\right)$ (Table 2, Fig. 2 and Fig. 3). Embolism resistant species would have evolved under arid conditions, adapting to function, survive and reproduce under more negative water potentials by investing in safety features to prevent embolism (Fig. 3 and Fig. S5). Both traits are evolutionarily correlated with water availability, confirming that they are crucial in maintaining hydraulic function and survival under severe drought-stress situations (Choat et al. 2018). We argue that a tight functional link between these two variables exists because a highly resistant xylem is needed to operate at low water potentials. The correlation between $\psi_{\min }$ and P50 holds even when environmental factors are controlled via their inclusion as fixed effects in our models, which suggests that $\psi_{\text {min }}$ is not merely a passive reflection of the environment, but an evolutionarily controlled characteristic driven partially by plant traits such as rooting depth, stomatal behaviour and leaf habit (Bhaskar \& Ackerly 2006; Martínez-Vilalta \& Garcia-Forner 2017). The positive relationship between HSM and water availability in Angiosperms (Fig. 1) suggests that these species usually adopt a riskier behaviour in drier environments, by approaching the minimum allowable water potential and potentially maximizing stomatal conductance and carbon gain at the expense of being closer to their physiological limit (Sperry et al. 1998). The opposite pattern occurs in Gymnosperms, which reach water potentials closer to their embolism resistance limit in more humid habitats. Gymnosperm species also present no relationship between $\psi_{\min }$ and water availability, suggesting fundamental differences in the way Angiosperms and Gymnosperms respond to arid conditions. These results are commensurate with the idea that many Gymnosperm species (particularly Pinus spp. ) strongly regulate their water potential (isohydric behaviour) via stomatal control or hydraulic disconnection from the soil hence avoiding extreme negative water potentials even under severe drought conditions (Plautet al. 2012; Poyatos et al. 2018). Nevertheless, the generality of this interpretation should be assessed in future works. 
The second trait integration we document involves transport efficiency and allocation $\left(K_{\mathrm{s}}-\mathrm{Hv}\right)$. Species with higher sapwood-specific conductivity can support higher leaf areas with relatively low investment in sapwood, thereby reducing the need for large cross-sectional areas and effectively leading to efficient and thin xylem cross sections for a given leaf area (Mencuccini et al. 2019). Both traits show significant evolutionary correlations with water availability (PC1) and with soil features (PC3), suggesting that these traits have been responding to the same environmental selective pressures by means of adaptation over evolutionary times (Fig. 2, Fig. 4 and Fig. S5). Again, however, the direct effect of the environment and major evolutionary affiliation on each trait is not enough to explain their evolutionary correlation (Table 2), suggesting that additional (genetic, physiological or developmental) processes are involved. The lack of relationship between $K_{1}$ and water availability is consistent with the reported coordination between $K_{\mathrm{s}}$ and $\mathrm{Hv}$, as a relatively conserved $K_{1}$ along environmental gradients suggests that plants are able to sustain similar transpiration rates by varying their carbon investment in sapwood or leaves in response to water scarcity (Fig. 1) (Manzoni et al. 2013).

No evolutionary correlation was found between xylem safety (P50) and efficiency $\left(K_{\mathrm{s}}\right)$. This is surprising given that the hypothesized mechanisms underlying this relationship involve direct structural trade-offs at the level of the inter-conduit pits (Sperry 2003) and the topology of the whole xylem network (Loepfe $e t$ al.2007; Martínez-Vilalta et al. 2012). The lack of strong evolutionary relationship between P50 and $K$ sindicates some level of decoupling between the structural determinants of safety and efficiency at the stem level (Gleason et al. 2016). At evolutionary timescales, our results likely reflect the fact that some species may present strategies that do not rely on maximizing both water transport efficiency and xylem safety, especially when water is not the most limiting resource and their survival does not depend on competition (slow economic strategy) (Reich 2014). Therefore, our result suggests that a xylem safety-efficiency trade-off may exert a minor contribution on lineage diversification. However, the detailed structural and physiological conditions that allow the independent evolution of these two traits at the plant level remain to be elucidated.

\section{Conclusion}

Hydraulic traits are under strong selective control and appear to be largely determined by deep-time evolutionary changes driven by adaptation to divergent environmental conditions. We have found evidence for the evolutionary integration of two adaptive modules, i.e., the xylem safety-exposure and the efficiency-allocation coordinations, but not for the hypothesized safety-efficiency trade-off. These evolutionary correlations, jointly with those reported with environmental conditions, point towards coordinated hydraulic adaptation to environmental pressures, limited by evolutionary constraints on trait evolution. More phylogenetically explicit studies of individual clades (including intraspecific genetic, anatomical and functional variation) under different environmental context will allow further characterization of the relationships among hydraulic traits worldwide. This would contribute to elucidate the adaptive capacity of plants to respond to the selective pressures caused by drought, which will be crucial under projected environmental forcing.

\section{Acknowledgments}

This work was supported by the Spanish government via competitive grants FUN2FUN (CGL2013-46808R) and DRESS (CGL2017-89149-C2-1-R). We would like to thank Jarrod Hadfield, Lucy Rowland, Toby Pennington, Paulo Bittencourt, Mario Marcos do Espirito Santo for fruitful discussions and insights on previous versions of the manuscript, as well as to Brendan Choat, Steven Jansen and Hui Liu for their previous work on data compilation. P.S.-M. acknowledges an FPU predoctoral fellowship from the Spanish Ministry of Science, Innovation and Universities (grant FPU18/04945). J.M.-V. benefited from an ICREA Academia award. R.A.S. is supported by a Newton International Fellowship from The Royal Society and by Conicyt PFCHA/Postdoctorado Becas Chile/2017 N 3140189 CONICYT PIA APOYO CCTE AFB170008. The data derived from the hydraulics database are partly an outcome from a working group funded by the Australian Research Council (ARC) through the Australia-New Zealand Research Network for Vegetation Function.

\section{Literature cited}


Ackerly, D. (2009). Conservatism and diversification of plant functional traits: Evolutionary rates versus phylogenetic signal. Proc. Natl. Acad. Sci. , 106, 19699-19706.

Ackerly, D.D., Dudley, Susan A, Sultan, S.E., Schmitt, J., Coleman, J.S., Linder, C.R., et al. (2000). The Evolution of Plant Ecophysiological Traits: Recent Advances and Future Directions.Bioscience, 50, 979.

Adams, H.D., Zeppel, M.J.B., Anderegg, W.R.L., Hartmann, H., Landhäusser, S.M., Tissue, D.T., et al. (2017). A multi-species synthesis of physiological mechanisms in drought-induced tree mortality.Nat. Ecol. Evol. , 1, 1285-1291.

Baker, T. R., Pennington, R. T., Dexter, K. G., Fine, P. V. A., Fortune-Hopkins, H., Honorio, E. N., et al . (2017). Maximising Synergy among Tropical Plant Systematists, Ecologists, and Evolutionary Biologists. Trends Ecol Evol. , 32(4), 258-267.

Bhaskar, R. \& Ackerly, D.D. (2006). Ecological relevance of minimum seasonal water potentials. Physiol. Plant. , 127, 353-359.

Butler, M.A. \& King, A.A. (2004). Phylogenetic comparative analysis: A modelling approach for adaptive evolution. Am. Nat. , 164, 683-695.

Cayuela, L., Granzow-de la Cerda, Í., Albuquerque, F.S. \& Golicher, D.J. (2012). Taxonstand: An r package for species names standardisation in vegetation databases. Methods Ecol. Evol. , 3, 1078-1083.

Choat, B., Brodribb, T., Brodersen, C., Duursma, R., López, R. \& Medlyn, B. (2018). Triggers of tree mortality under drought and forest mortality. Nature, 558, 531-539.

Choat, B., Jansen, S., Brodribb, T.J., Cochard, H., Delzon, S., Bhaskar, R., et al. (2012). Global convergence in the vulnerability of forests to drought. Nature, 491, 752-755.

Crisp, M.D., Arroyo, M.T.K., Cook, L.G., Gandolfo, M.A., Jordan, G.J., McGlone, M.S., et al. (2009). Phylogenetic biome conservatism on a global scale. Nature , 458, 754-756.

Crisp, M.D. \& Cook, L.G. (2012). Phylogenetic niche conservatism: What are the underlying evolutionary and ecological causes? New Phytol. , 196, 681-694.

Dexter, K. \& Chave, J. (2016). Evolutionary patterns of range size, abundance and species richness in Amazonian angiosperm trees.PeerJ , 4, e2402.

Dixon, H.H. (1914). Transpiration and the ascent of sap in plants. MacMillan, London, UK.

Etterson, J.R. \& Shaw, R.G. (2001). Constraint to adaptive evolution in response to global warming. Science , 294, 151-154.

Fick, S.E. \& Hijmans, R.J. (2017). WorldClim 2: new 1-km spatial resolution climate surfaces for global land areas. Int. J. Climatol. , 37, 4302-4315.

Gastauer, M. \& Meira Neto, J.A.A. (2017). Updated angiosperm family tree for analysing phylogenetic diversity and community structure.Acta Bot. Brasilica, 31, 191-198.

Gleason, S.M., Westoby, M., Jansen, S., Choat, B., Hacke, U.G., Pratt, R.B., et al. (2016). Weak trad eoff between xylem safety and xylem-specific hydraulic efficiency across the world's woody plant species. New Phytol. , 209, 123-136.

Goodwin, Z. A., Harris, D. J., Filer, D., Wood, J. R. I., \& Scotland, R. W. (2015). Widespread mistaken identity in tropical plant collections. Curr. Biol. , 25(22), R1066-R1067.

Hadfield, J.D. (2010). MCMCglmm for R. J. Stat. Softw. , 33(2), 1-22.

Hadfield, J.D. \& Nakagawa, S. (2010). General quantitative genetic methods for comparative biology: Phylogenies, taxonomies and multi-trait models for continuous and categorical characters. J. Evol. Biol. , 23, 494-508. 
Housworth, E.A., Martins, E.P. \& Lynch, M. (2004). The Phylogenetic Mixed Model. Am. Nat., 163, 84-96. Jacobsen, A.L., Pratt, R.B., Ewers, F.W. \& Davis, S.D. (2007). Cavitation resistance among 26 chaparral species of southern California.Ecol. Monogr. , 77, 99-115.

Jin, Y., \& Qian, H. (2019). V.PhyloMaker: An R package that can generate very large phylogenies for vascular plants. Ecography , 42(8), 1353-1359.

Katoh, K. \& Standley, D.M. (2013). MAFFT multiple sequence alignment software version 7: Improvements in performance and usability.Mol. Biol. Evol. , 30, 772-780.

Lamy, J.B., Delzon, S., Bouche, P.S., Alia, R., Vendramin, G.G., Cochard, H., et al. (2014). Limited genetic variability and phenotypic plasticity detected for cavitation resistance in a Mediterranean pine. New Phytol. , 201, 874-886.

Larter, M., Pfautsch, S., Domec, J.C., Trueba, S., Nagalingum, N. \& Delzon, S. (2017). Aridity drove the evolution of extreme embolism resistance and the radiation of conifer genus Callitris .New Phytol. , 215, $97-112$.

Leslie, A.B., Beaulieu, J., Holman, G., Campbell, C.S., Mei, W., Raubeson, L.R., et al. (2018). An overview of extant conifer evolution from the perspective of the fossil record. Am. J. Bot. , 105, 1531-1544.

Liu, H., Gleason, S.M., Hao, G., Hua, L., He, P., Goldstein, G., et al. (2019). Hydraulic traits are coordinated with maximum plant height at the global scale. Sci. Adv. , 5, eaav1332.

Loepfe, L., Martinez-Vilalta, J., Piñol, J. \& Mencuccini, M. (2007). The relevance of xylem network structure for plant hydraulic efficiency and safety. J. Theor. Biol., 247, 788-803.

Losos, J.B. (2008). Phylogenetic niche conservatism, phylogenetic signal and the relationship between phylogenetic relatedness and ecological similarity among species. Ecol. Lett. , 11, 995-1003.

Lynch, M. (1991). Methods for the analysis of comparative data in evolutionary biology. Evolution , 45, 1065-1079.

Magallón, S., Gómez-Acevedo, S., Sanchez-Reyes, L.L. \& Hernandez-Hernandez, T. (2015). A metacalibrated time-tree documents the early rise of flowering plant phylogenetic diversity. New Phytol. , 207, 437-453.

Maherali, H., Pockman, W.T. \& Jackson, R.B. (2004). Adaptive variation in the vulnerability of woody plants to xylem cavitation.Ecology , 85, 2184-2199.

Manzoni, S., Vico, G., Katul, G., Palmroth, S., Jackson, R.B. \& Porporato, A. (2013). Hydraulic limits on maximum plant transpiration and the emergence of the safety-efficiency trade-off. New Phytol. , 198, $169-178$.

Martinez-Vilalta, J. \& Garcia-Forner, N. (2017). Water potential regulation, stomatal behaviour and hydraulic transport under drought: deconstructing the iso/anisohydric concept. Plant Cell Environ. , 40, 962-976.

Martinez-Vilalta, J., Mencuccini, M., Alvarez, X., Camacho, J., Loepfe, L. \& Pinol, J. (2012). Spatial distribution and packing of xylem conduits. Am. J. Bot. , 99, 1189-1196.

Mencuccini, M., Rosas, T., Rowland, L., Choat, B., Cornelissen, H., Jansen, S., et al. (2019). Leaf economics and plant hydraulics drive leaf: wood area ratios. New Phytol . 224: 1544-1556.

Nakagawa, S. \& Schielzeth, H. (2013). A general and simple method for obtaining R2 from generalized linear mixed-effects models. Methods Ecol. Evol. , 4, 133-142.

Neves, D.M., Dexter, K.G., Baker, T.R., Coelho de Souza, F., Oliveira-Filho, A.T., Queiroz, L.P., et al. (2020). Evolutionary diversity in tropical tree communities peaks at intermediate precipitation. Sci. Rep. , 10, 1188. 
Niklas, K.J. (2007). Maximum plant height and the biophysical factors that limit it. Tree Physiol. , 27, 433-440.

Pennell, M.W., FitzJohn, R.G. \& Cornwell, W.K. (2016). A simple approach for maximizing the overlap of phylogenetic and comparative data. Methods Ecol. Evol. , 7, 751-758.

Plaut, J.A., Yepez, E.A., Hill, J., Pangle, R., Sperry, J.S., Pockman, W.T., et al. (2012). Hydraulic limits preceding mortality in a pinon-juniper woodland under experimental drought. Plant, Cell Environ. , 35, $1601-1617$.

Poyatos, R., Aguade, D. \& Martinez-Vilalta, J. (2018). Below-ground hydraulic constraints during droughtinduced decline in Scots pine.Ann. For. Sci. , 75.

Ran, J.H., Shen, T.T., Wang, M.M. \& Wang, X.Q. (2018). Phylogenomics resolves the deep phylogeny of seed plants and indicates partial convergent or homoplastic evolution between Gnetales and angiosperms.Proc. R. Soc. B Biol. Sci., 285.

R Core Team (2017). R: A language and environment for statistical computing. R Foundation for Statistical Computing, Vienna, Austria.

Reich, P.B. (2014). The world-wide "fast-slow" plant economics spectrum: A traits manifesto. J. Ecol. , $102,275-301$.

Revell, L.J. (2013). Two new graphical methods for mapping trait evolution on phylogenies. Methods Ecol. Evol. , 4, 754-759.

Revell, L.J., Harmon, L.J. \& Collar, D.C. (2008). Phylogenetic signal, evolutionary process, and rate. Syst. Biol. , 57, 591-601.

Ribeiro, E., Kilibarda, M., Geng, X., MacMillan, R.A., Blagotić, A., Ruiperez Gonzalez, M., et al. (2017). SoilGrids250m: Global gridded soil information based on machine learning. PLoS One , 12(2): e0169748.

Schluter, D., Price, T., Mooers, A.T. \& Ludwig, D. (1997).Evolution , 51(6), 1699-1711.

Segovia, R. A., Pennington, T., Baker, T., Coelho de Souza, F., Neves, D., Davis, C. et al. (2020). Freezing and water availability structure the evolutionary diversity of trees across the Americas.Sci. Adv. , in press (accepted).

Smith, S. A., \& Brown, J. W. (2018). Constructing a broadly inclusive seed plant phylogeny. Am. J. Bot. , 105(3), 302-314.

Smith, S.A. \& O'Meara, B.C. (2012). TreePL: Divergence time estimation using penalized likelihood for large phylogenies. Bioinformatics , 28, 2689-2690.

Sperry, J.S. (2003). Evolution of Water Transport and Xylem Structure.Int. J. Plant Sci. , 164, S115-S127.

Sperry, J.S., Adler, F.R., Campbell, G.S. \& Comstock, J.S. (1998). Hydraulic limitation of flux and pressure in the soil-plant continuum: results from a model. Plant Cell Environ. , 21, 347-359.

Stamatakis, A., Hoover, P. \& Rougemont, J. (2008). A rapid bootstrap algorithm for the RAxML web servers. Syst. Biol. , 57, 758-771.

Tank, D.C., Eastman, J.M., Pennell, M.W., Soltis, P.S., Soltis, D.E., Hinchliff, C.E., et al. (2015). Nested radiations and the pulse of angiosperm diversification: increased diversification rates often follow whole genome duplications. New Phytol. , 207, 454-467.

Trabucco, A. \& Zomer, R.J. (2018). Global Aridity Index and Potential Evapotranspiration (ET0) Climate Database v2. CGIAR Consort. Spat. Inf. , 10.

Tyree M.T. \& Zimmermann M.H. (2002). Xylem Structure and the Ascent of Sap. Springer, New York. 
Villemereuil, P. \& Nakagawa, S. (2014). General Quantitative Genetic Methods for Comparative Biology. In: (Modern Phylogenetic Comparative Methods and Their Application in Evolutionary Biology: Concepts and Practice ), \{[Garamszegi, L.Z.]\}. Springer, New York, USA. 287-303.

Willson, C.J., Manos, P.S. \& Jackson, R.B. (2008). Hydraulic traits are influenced by phylogenetic history in the drought-resistant, invasive genus Juniperus (Cupressaceae). Am. J. Bot. , 95, 299-314.

Zanne, A.E., Tank, D.C., Cornwell, W.K., Eastman, J.M., Smith, S.A., Fitzjohn, R.G., et al. (2014). Three keys to the radiation of angiosperms into freezing environments. Nature , 506, 89-92.

Zheng, J., Zhao, X., Morris, H. \& Jansen, S. (2019). Phylogeny Best Explains Latitudinal Patterns of Xylem Tissue Fractions for Woody Angiosperm Species Across China. Front. Plant Sci. , 10: 556.

Tables

Table 1. Variance partitioning for six hydraulic traits and three environmental principal components. Legend: N: number of species used in each case (for which both phylogenetic and hydraulic data were available), phylogenetic variance $(\lambda)$, non-phylogenetic inter-generic variance $(\gamma)$ and non-phylogenetic intrageneric variance plus measurement error $(\rho)$. Mean and lower and upper $95 \%$ credible intervals (HDP) are shown for each component.

\begin{tabular}{lllllllllll}
\hline variable & $\mathrm{N}$ & $\lambda$ & Lower HPD & Upper HPD & $\gamma$ & Lower HDP & Upper HDP & $\rho$ & Lower HDP & Uppe \\
\hline $\log (-|\mathrm{P} 50|)$ & 869 & 0.484 & 0.305 & 0.697 & 0.225 & 0.085 & 0.360 & 0.291 & 0.205 & 0.368 \\
$\log \left(\left|\psi_{\min }\right|\right)$ & 541 & 0.745 & 0.572 & 0.874 & 0.066 & 0.000 & 0.179 & 0.189 & 0.129 & 0.273 \\
$\log \left(K_{\mathrm{s}}\right)$ & 1023 & 0.515 & 0.363 & 0.680 & 0.086 & 0.000 & 0.174 & 0.399 & 0.303 & 0.493 \\
$\log (\mathrm{Hv})$ & 1270 & 0.446 & 0.291 & 0.594 & 0.191 & 0.097 & 0.294 & 0.363 & 0.276 & 0.449 \\
$\mathrm{HSM}$ & 326 & 0.488 & 0.252 & 0.723 & 0.068 & 0.000 & 0.217 & 0.444 & 0.262 & 0.623 \\
$\log \left(K_{\mathrm{l}}\right)$ & 824 & 0.432 & 0.244 & 0.592 & 0.036 & 0.000 & 0.113 & 0.532 & 0.399 & 0.675 \\
PC1 & 1971 & 0.820 & 0.767 & 0.870 & 0.063 & 0.030 & 0.099 & 0.117 & 0.093 & 0.139 \\
PC2 & 1971 & 0.686 & 0.599 & 0.766 & 0.028 & 0.000 & 0.069 & 0.286 & 0.230 & 0.341 \\
PC3 & 1971 & 0.841 & 0.798 & 0.876 & 0.007 & 0.000 & 0.027 & 0.152 & 0.124 & 0.182 \\
\hline
\end{tabular}

Table 2. Evolutionary correlations and variance distribution of each pair of hydraulic traits reported in the best models accounting for fixed effects. Legend: N, number of species employed (for which phylogenetic data and values for the two traits involved were available); Phylogenetic $(\lambda)$, Inter-generic $(\gamma)$ and Intra-generic $(\rho)$ account for the phylogenetic, inter-generic and intra-generic plus measurement error variance components; $\mathrm{E}$. Correlation, evolutionary correlation for the pair of traits; Intercept., Spermatophyte ancestral value when major evolutionary affiliation was not included and Angiosperm ancestral value when included; PC1, PC2 and PC3, environmental fixed effects; Gym. Int., gymnosperm ancestral value; PC1: gym, PC2: gym and PC3: gym, environmental fixed effects for gymnosperms (when interaction between environment and major evolutionary affiliation was included). Signif. codes (referring as a statistical difference from zero for each parameter): ‘***': $\mathrm{P}<0.001$; '**': $\mathrm{P}<0.01$; $^{(*)}: \mathrm{P}<0.05^{\prime}{ }^{\prime}: \mathrm{P}<0.1$ ' $: \mathrm{P}>0.1$. When significance codes are not shown, $95 \%$ credible intervals are displayed in parenthesis.

\begin{tabular}{|c|c|c|c|c|c|c|c|c|c|c|c|}
\hline & $\mathrm{N}$ & $\begin{array}{l}\text { Phyloge } \\
(\lambda)\end{array}$ & $\begin{array}{l}\text { efinter- } \\
\text { generic }(\gamma\end{array}$ & $\begin{array}{l}\text { Intra- } \\
\text { generic } \\
(p)\end{array}$ & $\begin{array}{l}\text { E. } \\
\text { Correlati }\end{array}$ & oIntercept & PC1 & $\mathrm{PC} 2$ & PC3 & $\begin{array}{l}\text { Gym. } \\
\text { Int }\end{array}$ & PC1:gym \\
\hline $\log \left(K_{\mathrm{S}}\right)$ & 601 & $\begin{array}{l}0.430 \\
(0.246, \\
0.609)\end{array}$ & $\begin{array}{l}0.166(0.0 \\
0.284)\end{array}$ & $\begin{array}{l}60,404(0.2 \\
0.519)\end{array}$ & $\begin{array}{c}940.112 \\
(-0.509 \\
0.293)\end{array}$ & -0.046 & $0.101^{* * *}$ & -0.050 & $\begin{array}{l}- \\
0.129^{* * *}\end{array}$ & & \\
\hline
\end{tabular}




\begin{tabular}{|c|c|c|c|c|c|c|c|c|c|c|}
\hline $\mathrm{N}$ & $\begin{array}{l}\text { Phyloge } \\
(\lambda)\end{array}$ & $\begin{array}{l}\text { efinter- } \\
\text { generic }(\gamma\end{array}$ & $\begin{array}{l}\text { Intra- } \\
\text { generic } \\
(\rho)\end{array}$ & $\begin{array}{l}\text { E. } \\
\text { Correlati }\end{array}$ & iomntercept & $\mathrm{PC} 1$ & $\mathrm{PC} 2$ & PC3 & $\begin{array}{l}\text { Gym. } \\
\text { Int }\end{array}$ & PC1:gym \\
\hline $\log (|\mathrm{P} 50|)$ & $\begin{array}{l}0.343 \\
(0.212, \\
0.489)\end{array}$ & $\begin{array}{l}0.181(0.0 \\
0.265)\end{array}$ & $\begin{array}{c}99,476(0 . \\
0.571)\end{array}$ & & $1.170^{* * *}$ & $\overline{-}-045^{* * *}$ & $-\overline{0}$ & 0.037 . & & \\
\hline $\log \left(K_{\mathrm{s}}\right) \quad 773$ & $\begin{array}{l}0.399(0 . \\
0.564)\end{array}$ & $\begin{array}{c}2 \oplus, 156(0.0 \\
0.247)\end{array}$ & $\begin{array}{l}50,446(0 . \\
0.563)\end{array}$ & $\begin{array}{c}370.439 \\
(-0.805 \\
-0.030)\end{array}$ & -0.069 & $0.154^{* * *}$ & -0.052 & $-\overline{0.090^{* *}}$ & & \\
\hline $\log (\mathrm{Hv})$ & $\begin{array}{l}0.230 \\
(0.104, \\
0.370)\end{array}$ & $\begin{array}{l}0.331(0.1 \\
0.448)\end{array}$ & $\begin{array}{c}90,439(0 . \\
0.555)\end{array}$ & & 0.582 & $-\overline{0.212 * * *}$ & 0.015 & $0.100^{* * *}$ & & \\
\hline $\log \left(K_{\mathrm{s}}\right) \quad 422$ & $\begin{array}{l}0.285 \\
(0.078, \\
0.493)\end{array}$ & $\begin{array}{l}0.282(0.1 \\
0.441)\end{array}$ & $\begin{array}{c}20,433(0 . \\
0.569)\end{array}$ & $\begin{array}{c}9 \notin, 061 \\
(-0.499 \\
0.572)\end{array}$ & 0.513 & $0.196^{* * *}$ & -0.059 & -0.089 & -1.091 & -0.046 \\
\hline $\log \left(\left|\psi_{\min }\right|\right)$ & $\begin{array}{l}0.435 \\
(0.220, \\
0.623)\end{array}$ & $\begin{array}{l}0.284(0.1 \\
0.433)\end{array}$ & $\begin{array}{c}3 \emptyset, 282(0 . \\
0.382)\end{array}$ & & 0.406 & $-\overline{0.162 * * *}$ & $-\overline{0.053^{* *}}$ & $-\overline{0}$ & 0.639 & 0.046 \\
\hline $\log (|\mathrm{P} 50|) 466$ & $\begin{array}{l}0.379 \\
(0.231 \\
0.529)\end{array}$ & $\begin{array}{l}0.166(0.0 \\
0.250)\end{array}$ & $\begin{array}{c}80,455(0 . \\
0.563)\end{array}$ & $\begin{array}{c}46,060 \\
(-0.384 \\
0.537)\end{array}$ & $1.153^{* * *}$ & $-\overline{0.077^{* * *}}$ & -0.039 & 0.024 & & \\
\hline $\log (\mathrm{Hv})$ & $\begin{array}{l}0.232 \\
(0.096, \\
0.382)\end{array}$ & $\begin{array}{l}0.307(0.1 \\
0.442)\end{array}$ & $\begin{array}{c}68,462(0 . \\
0.589)\end{array}$ & & $0.617^{* *}$ & $-\overline{0.237 * * *}$ & $-\overline{0}$ & $0.135^{* * *}$ & & \\
\hline $\log (\mathrm{Hv}) \quad 374$ & $\begin{array}{l}0.242 \\
(0.066, \\
0.435)\end{array}$ & $\begin{array}{l}0.457(0.2 \\
0.620)\end{array}$ & $\begin{array}{c}70,301(0 . \\
0.416)\end{array}$ & $\begin{array}{c}030.176 \\
(-0.691 \\
0.387)\end{array}$ & 0.606 & $\overline{0}-272^{* * *}$ & 0.048 & 0.042 & 0.328 & -0.140 \\
\hline $\log \left(\left|\psi_{\min }\right|\right)$ & $\begin{array}{l}0.416(0 . \\
0.609)\end{array}$ & $\begin{array}{c}18,247(0.1 \\
0.367)\end{array}$ & $\begin{array}{c}08,336(0 . \\
0.451)\end{array}$ & & 0.461 & $-\overline{0.175^{* * *}}$ & $\begin{array}{l}- \\
0.044^{* *}\end{array}$ & $\begin{array}{l}- \\
0.053\end{array}$ & 0.557 & 0.025 \\
\hline $\log \left(\left|\psi_{\min }\right| \$ 20\right.$ & $\begin{array}{l}0.451 \\
(0.235, \\
0.649)\end{array}$ & $\begin{array}{l}0.260(0.1 \\
0.415)\end{array}$ & $\begin{array}{c}10,289(0 . \\
0.401)\end{array}$ & $\begin{array}{c}90,587 \\
(0.253 \\
0.879)\end{array}$ & 0.494 & $-\overline{0.161 * * *}$ & -0.050 & 0.022 & 0.612 & 0.035 \\
\hline $\log (|\mathrm{P} 50|)$ & $\begin{array}{l}0.400 \\
(0.199, \\
0.609)\end{array}$ & $\begin{array}{l}0.326(0.1 \\
0.487)\end{array}$ & $\begin{array}{c}60,274(0 . \\
0.373)\end{array}$ & & 0.775 & $-\overline{0.064^{* * *}}$ & 0.014 & $0.087^{* *}$ & 0.858 & $\begin{array}{l}- \\
0.020\end{array}$ \\
\hline
\end{tabular}

\section{Figure captions}

Figure 1. Trait-environment relationships. Relationships between environmental principal components (PC1 to PC3) and hydraulic traits (log-transformed absolute values) accounting for the phylogenetic structure of the last. The best model for each case is displayed, showing the Spermatophyte level relationship (black) or the Angiosperms and Gymnosperms relationships (red and blue, respectively) when statistically different. Grey dashed lines represent the regression line at the Spermatophyte level without accounting for the phylogenetic structure. Statistically significant $(\mathrm{p}<0.05)$ regression slopes are displayed in bold following the same colour code. Signif. codes: '***': $\mathrm{P}<0.001$; $^{(* *)}: \mathrm{P}<0.01$; '*': $\mathrm{P}<0.05$ ' $: \mathrm{P}<0.1^{\text {' }}$ ': $\mathrm{P}>0.1$. $\mathrm{R}^{2} \mathrm{~m}$ is the variance explained by the fixed effects and $\mathrm{R}^{2} \mathrm{c}$ by the fixed and random effects for the phylogenetic mixed models.

Figure 2. Evolutionary correlations between hydraulic traits and between traits and environmental variables (environmental variables represent orthogonal PC axes and as such are not correlated). Lines represent significant evolutionary correlations, with the thickness of the line proportional to the strength of the cor- 
relation coefficient (also given on the same line). Light red lines represent negative relationships, dark blue one's indicate positive relationships. Significant correlations coefficients between traits when excluding environmental effects and evolutionary affiliation as fixed effects are shown in italics, and significant correlation coefficients between traits obtained from best model in each case (i.e., including fixed effects as reported in Table 2) are shown in bold. Dashed line represents evolutionary correlation only significant when environmental effects and major evolutionary affiliation were not considered. Pie charts represent phylogenetic signal (dark), inter-genetic (medium) and intra-genetic (light) variances reported in table 1 (i.e., calculated using the maximum number of observations for each case).

Figure 3. Phylogenetic reconstruction of drought exposure and embolism resistance under a Brownian motion model of evolution. Families with more than one genus are presented and some of the most important families are highlighted in bold. Gymnosperm families are displayed in grey and Angiosperm ones in black.

Figure 4. Phylogenetic reconstruction of hydraulic efficiency and sapwood/leaf allocation (Huber value). Families with more than one genus are presented and some of the most important families are highlighted in bold. Gymnosperm families are displayed in grey and Angiosperm ones in black.

Figure 1 

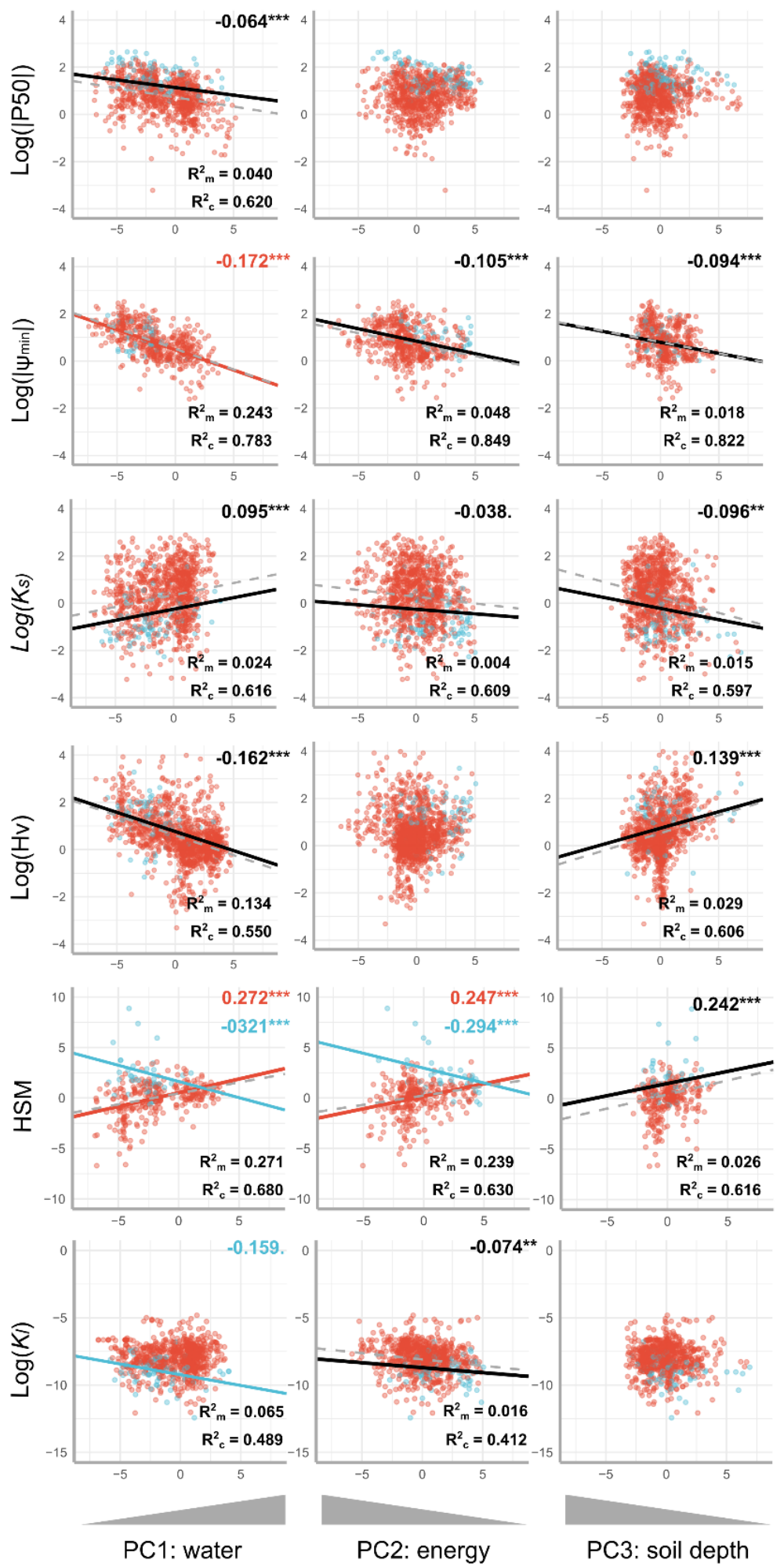

PC1: water

PC2: energy

PC3: soil depth

Angiosperms - Spermatophyta
Gymnosperms - - Non-Phylo Spermatophyta 
Figure 2

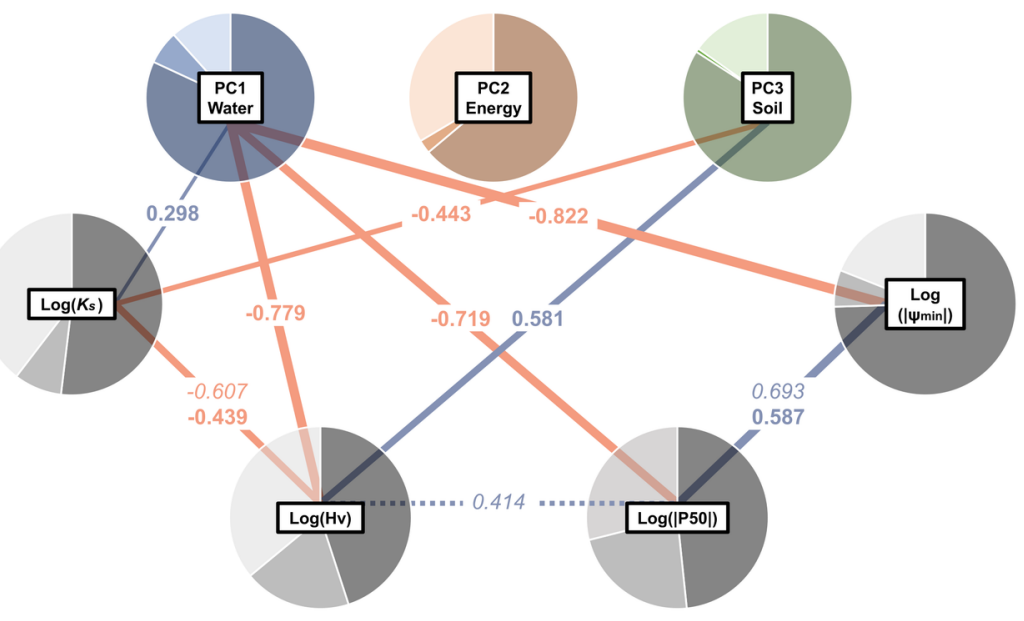

Figure 3 


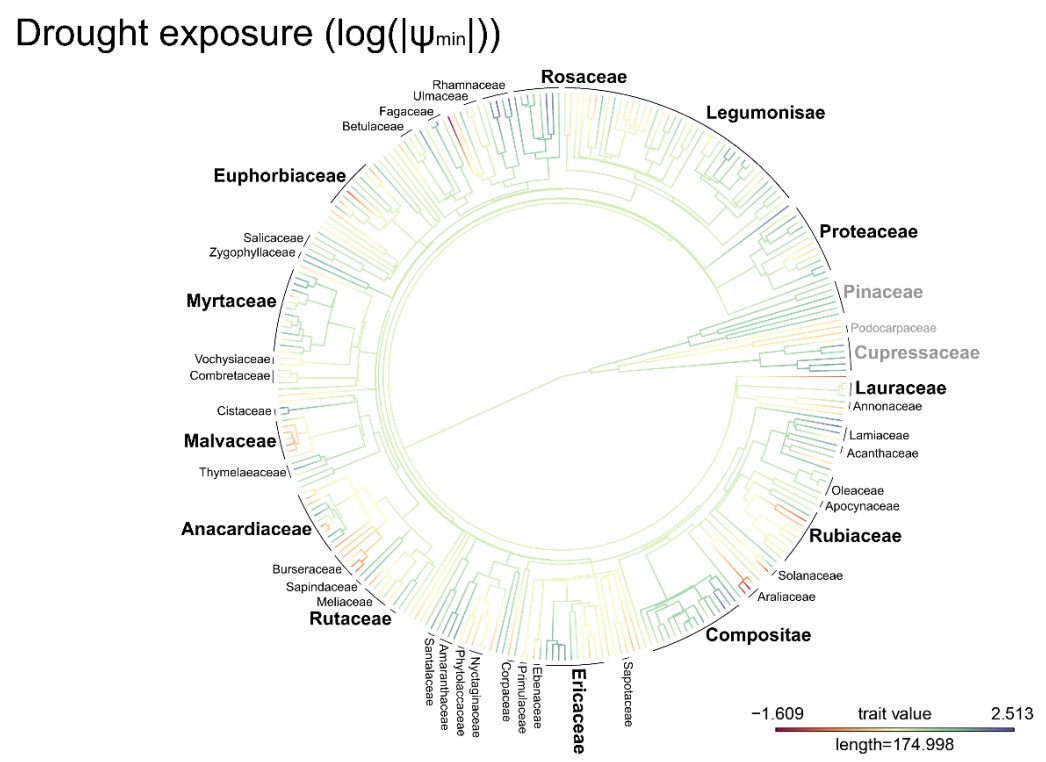

\section{Embolism resistance $(\log (|\mathrm{P} 50|))$}

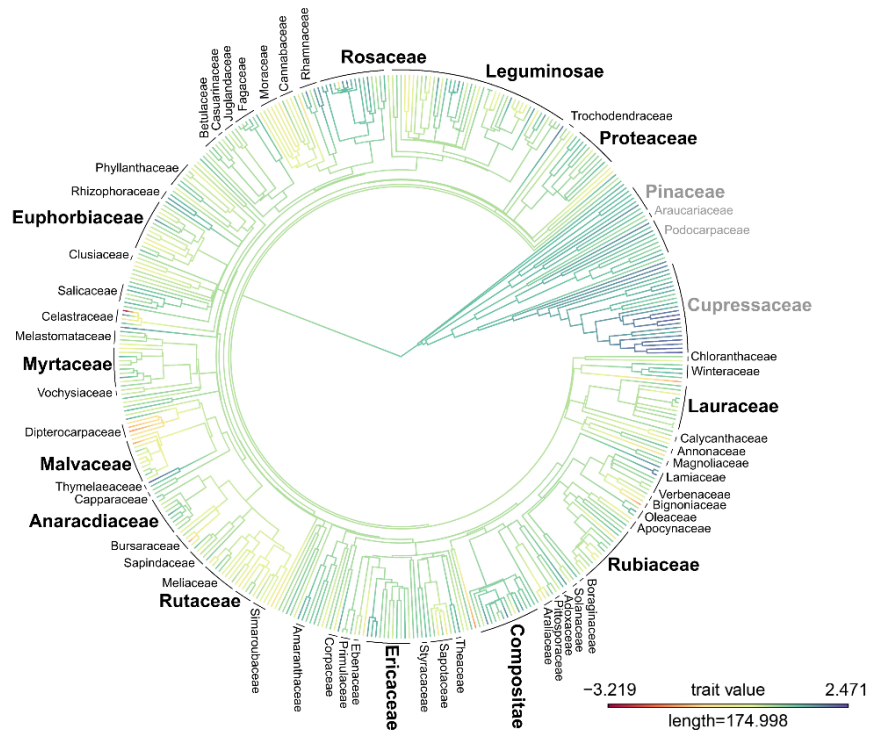

Figure 4 


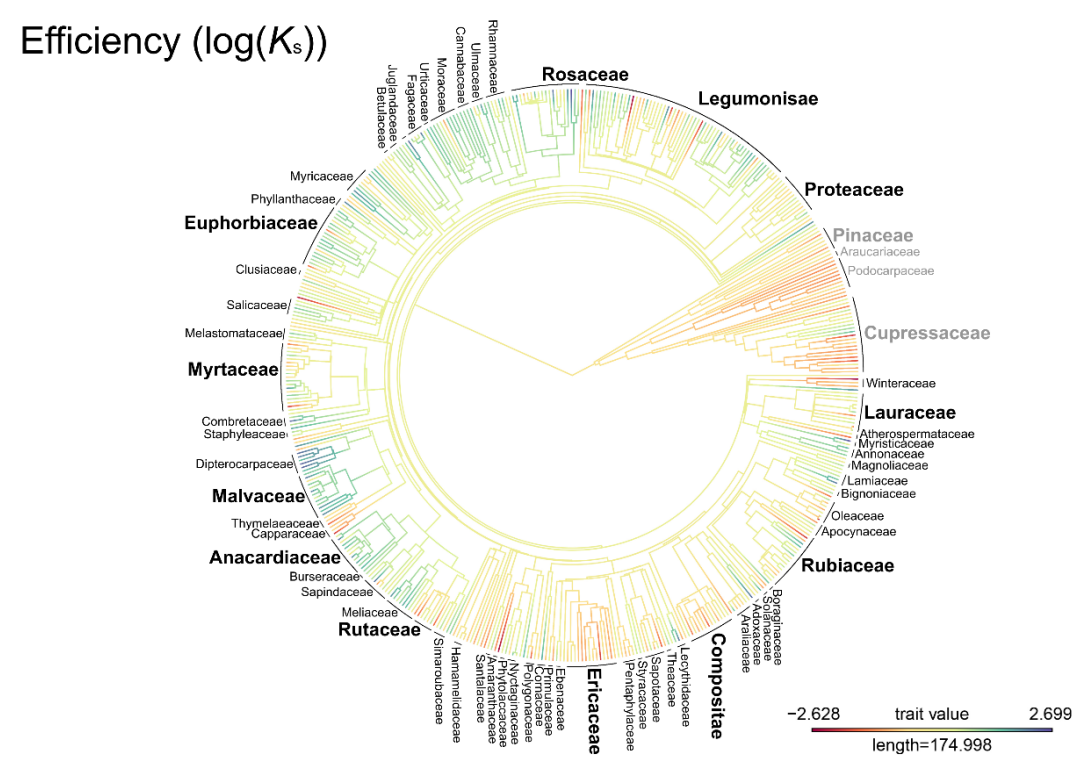

Sapwood/leaf allocation $(\log (\mathrm{Hv}))$

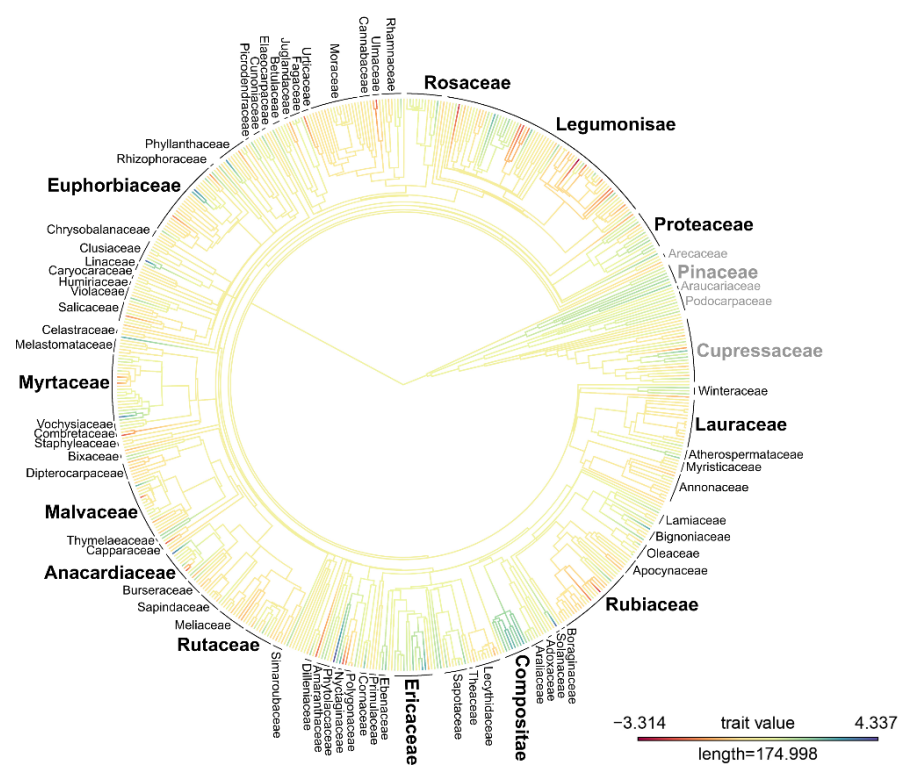

\section{Supporting information}

Figure S1. Hypotheses.

Double-headed arrows represent the hypothesized adaptive module, or evolutionary coordination, involving key hydraulic traits. Blue lines represent hypothetical positive relationships between traits, and red lines hypothetical negative ones. Black curved arrows represent traits phylogenetic variance. Each hypothesized coordination between traits is also encircled using long dashed lines and labelled accordingly. 


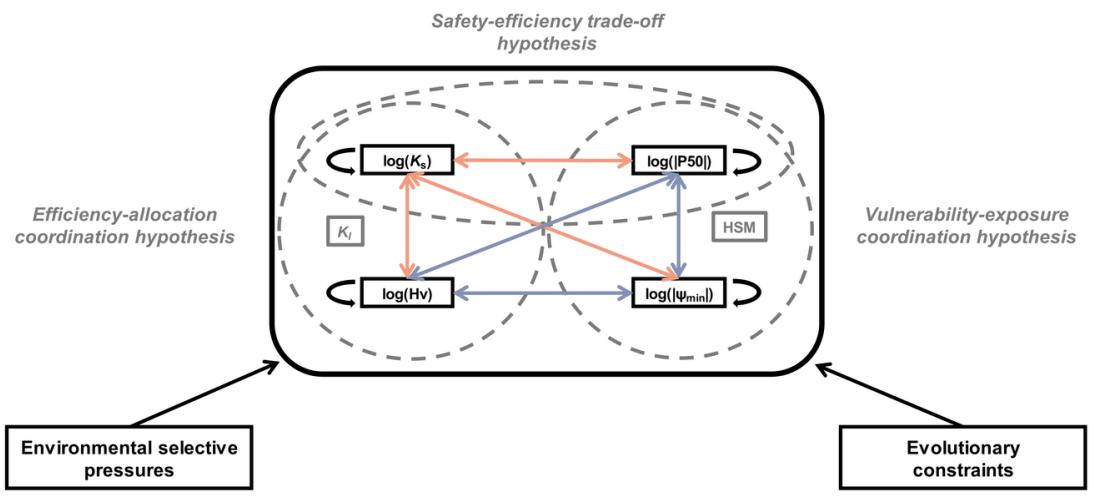

Figure S2. Geographic distribution of the three main environmental principal components.

Species-mean coordinates are plotted here for each species. Thus, some coordinates fall into the sea (presumably species present in both Palearctic and Nearctic). However, note that environmental variables were calculated for each occurrence of each species separately and then averaged to species level. 


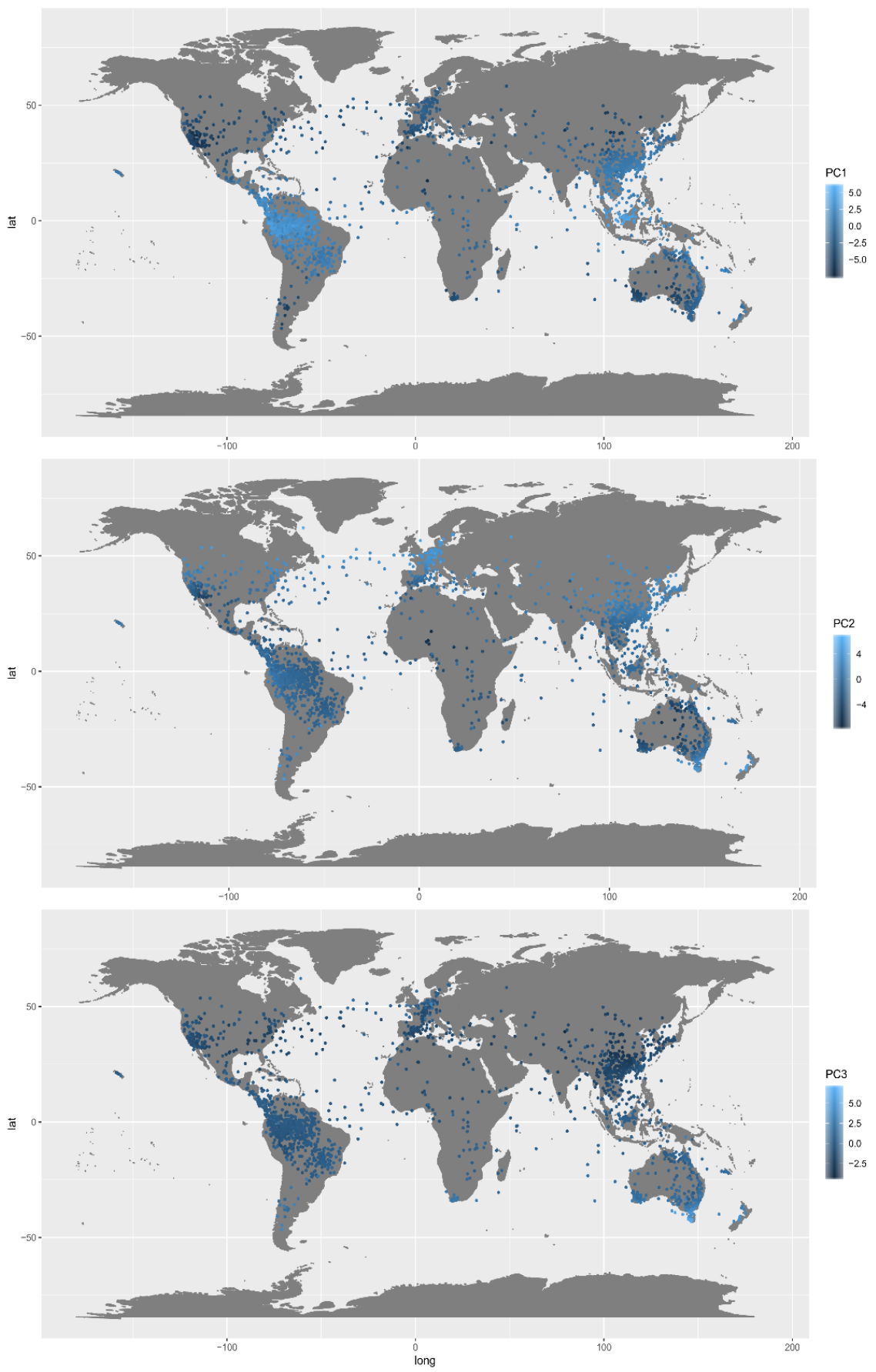

Figure S3. Whittaker diagrams.

Whittaker diagrams for all observations available (once matched with the phylogeny) and observations used for each one of the evolutionary correlations calculation (which has been restricted to those species with complete observations for the two traits and with genus-level phylogenetic information available). 


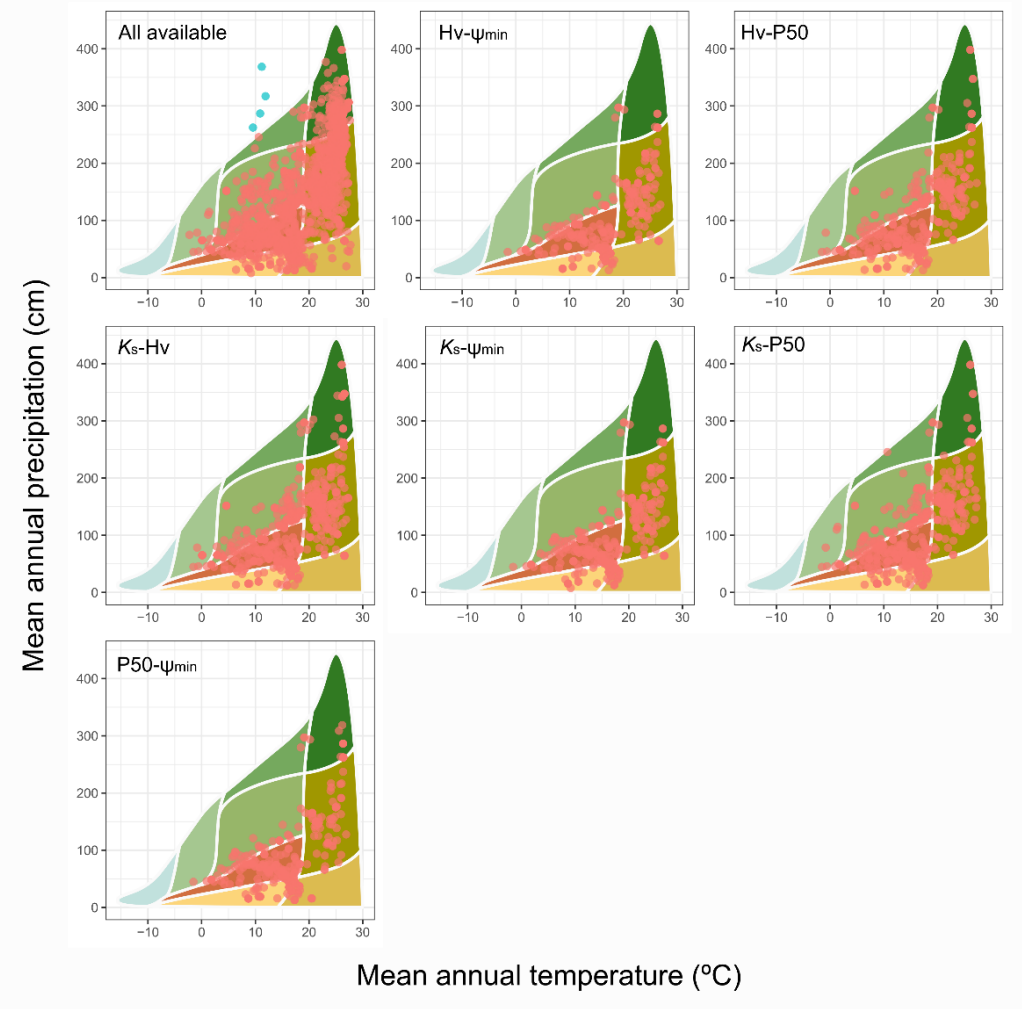

Figure S4. PCA biplot environment-hydraulic relationships.

a), b): PCA biplots showing the contributions of environmental variables to the first two principal components, PC1 and PC2 (a) and to PC1 and PC3 (b), colouring species as Angiosperms (red circles) or Gymnosperms (light blue triangles). See Table S1 for variable abbreviations.

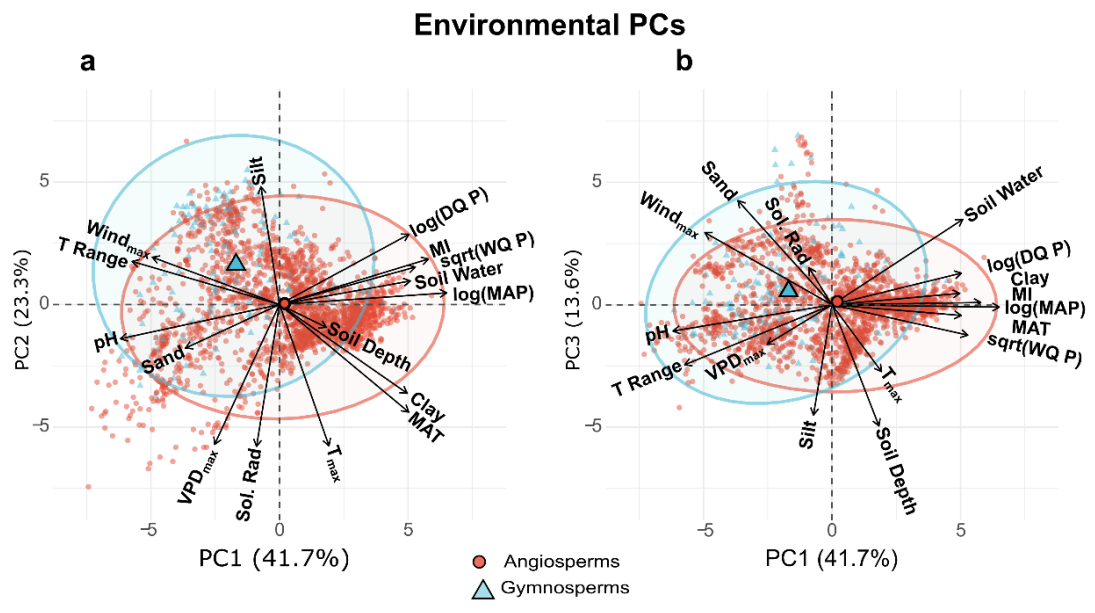

Figure S5. Phylogenetic reconstruction of the three environmental principal components under a Brownian motion model of evolution. 
Families with more than one genus are presented and some of the most important families are highlighted in bold. Gymnosperm families are displayed in grey and Angiosperm ones in black.

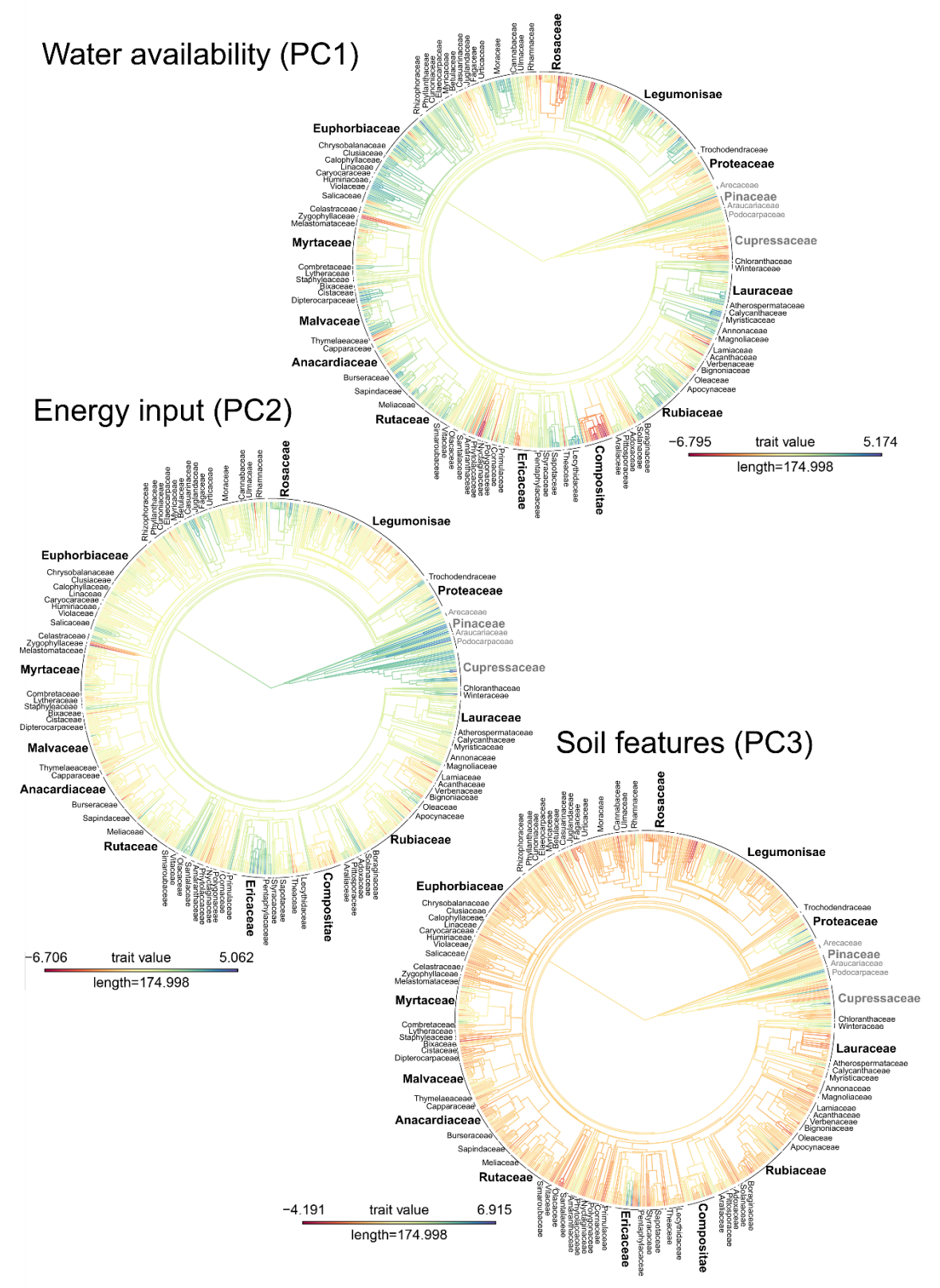

Figure S6. Phylogenetic reconstruction of Hydraulic Safety Margin (HSM) and leaf-specific hydraulic conductivity $\left(\log \left(K_{l}\right)\right)$ under a Brownian motion model of evolution.

Families with more than one genus are presented and some of the most important families are highlighted in bold. Gymnosperm families are displayed in grey and Angiosperm ones in black. 


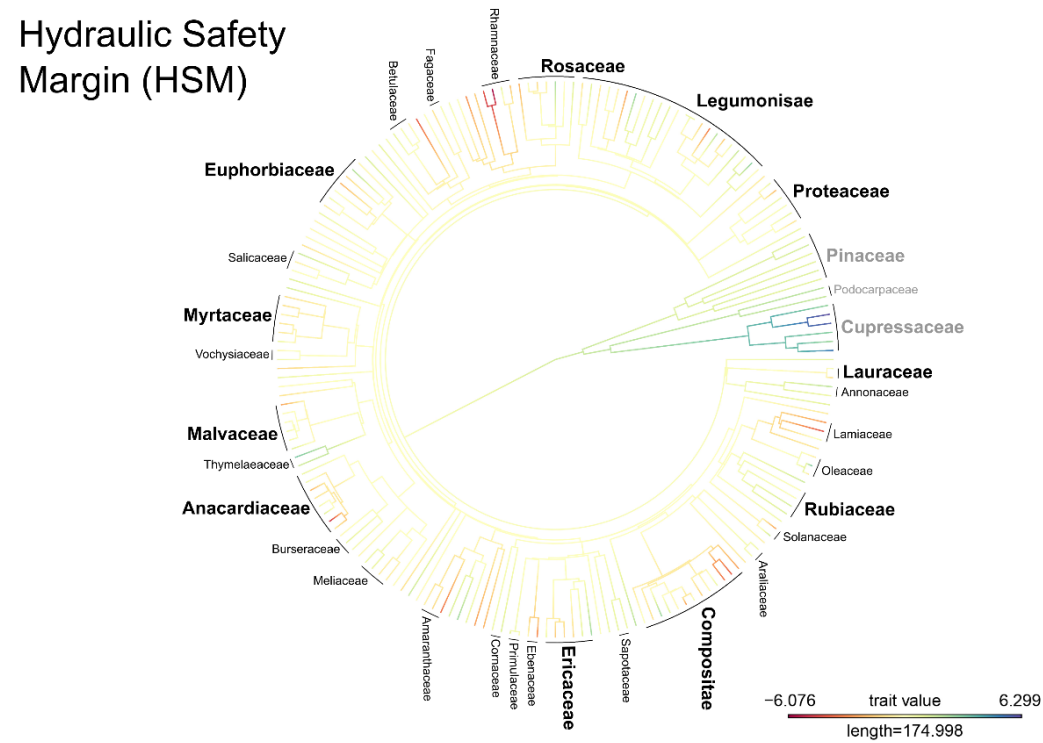

Leaf specific conductivity $\left(\log \left(K_{1}\right)\right)$

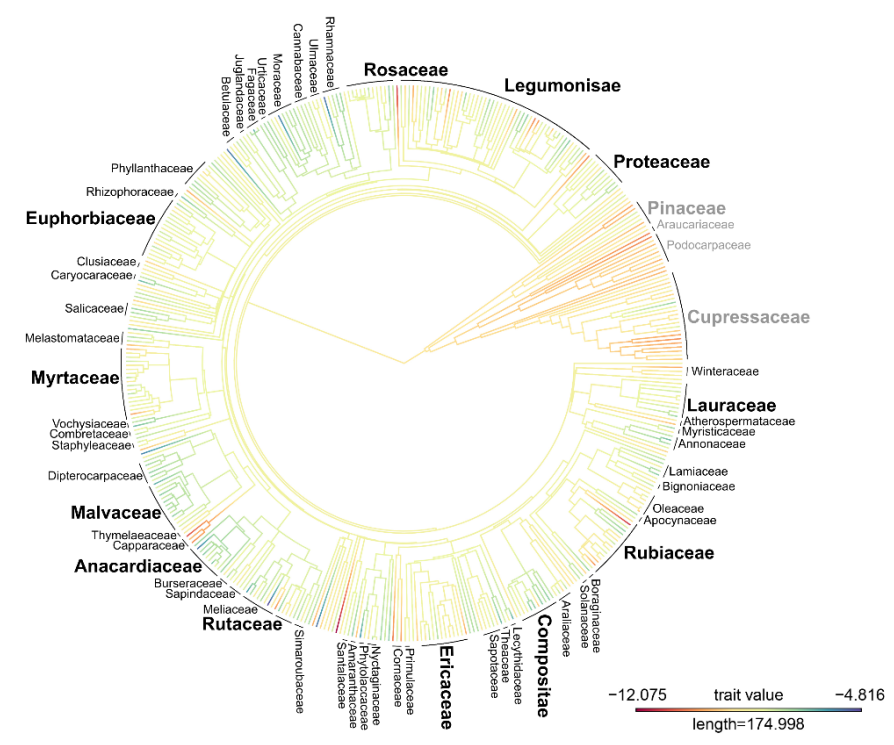

Table S1. Number of observations variables abbreviation and transformations.

Environmental variable and hydraulic traits nomenclature and number of whole dataset and major evolutionary affiliation observations. In the abbreviation column transformations are specified, when implemented.

Variable

Absolute Depth to Bed Rock

Aridity Index (Moisture index)

Clay content in percentage measured at $60 \mathrm{~cm}$

Maximum Temperature Warmest month

Maximum Vapour Pressure Deficit

Maximum Wind

\begin{tabular}{llll}
\hline Abbreviation & Observations & Angiosperms & Gymno \\
Soil Depth & 2001 & 1867 & 134 \\
MI & 2064 & 1917 & 147 \\
Clay & 2001 & 1867 & 134 \\
$\mathrm{~T}_{\max }$ & 2001 & 1867 & 134 \\
VPD $_{\max }$ & 2001 & 1867 & 134 \\
wind $_{\max }$ & 2001 & 1867 & 134
\end{tabular}


Mean Annual Precipitation (log. transf.)

Mean Annual Temperature

$\mathrm{pH}$ measured at $200 \mathrm{~cm}$

Precipitation Driest Quarter (log. transf.)

Precipitation Warmest month (sqrt. transf.)

Sand content in percentage measured at $60 \mathrm{~cm}$

Silt content in percentage measured at $60 \mathrm{~cm}$

Soil Water Content at $200 \mathrm{~cm}$

Solar radiation

Temperature Range

Potential at the $50 \%$ conductivity loss (absolute value log. transf.)

Minimum water potential (absolute value log. transf.)

Specific hydraulic conductivity (log. transf.)

Huber value (sapwood area: leaf area ratio) (log. transf.)

Hydraulic Safety Margin

Leaf-specific hydraulic conductivity (log. transf.)

\begin{tabular}{llll} 
MAP & 2067 & 1920 & 147 \\
MAT & 2067 & 1920 & 147 \\
pH & 2001 & 1867 & 134 \\
DQ P & 2001 & 1867 & 134 \\
WQ P & 2001 & 1867 & 134 \\
Sand & 2001 & 1867 & 134 \\
Silt & 2001 & 1867 & 134 \\
Soil Water & 2001 & 1867 & 134 \\
Sol.Rad & 2001 & 1867 & 134 \\
TRange & 2001 & 1867 & 134 \\
P50 & 898 & 772 & 126 \\
$\psi_{\text {min }}$ & 551 & 503 & 48 \\
$K_{\mathrm{s}}$ & 1050 & 949 & 101 \\
Hv & 1299 & 1223 & 76 \\
HSM & 334 & 292 & 42 \\
$K_{1}$ & 845 & 767 & 78 \\
\hline
\end{tabular}

Table S2. Contribution of environmental variables to the three environmental principal components.

The highest contribution is highlighted for each variable. See table S1 for meaning of abbreviations.

\begin{tabular}{llll}
\hline Variable & Dim.1 $(41.7 \%)$ & Dim.2 $(23.3 \%)$ & Dim.3 $(13.6 \%)$ \\
Sqrt(WQ P) & $\mathbf{8 . 4 9 2}$ & 1.280 & 1.436 \\
T max & 1.126 & $\mathbf{1 8 . 1 9 9}$ & 6.956 \\
Log(MAP) & $\mathbf{1 2 . 8 8 0}$ & 0.124 & 0.008 \\
Log(DQ P) & $\mathbf{7 . 7 0 9}$ & 4.489 & 1.571 \\
MAT & 7.689 & $\mathbf{1 0 . 5 4 1}$ & 0.176 \\
MI & $\mathbf{1 0 . 2 0 5}$ & 1.890 & 0.009 \\
Sol. Rad & 0.242 & $\mathbf{1 8 . 3 2 7}$ & 2.128 \\
windMax & 7.446 & 2.038 & $\mathbf{8 . 1 5 8}$ \\
VPDMax & 1.939 & $\mathbf{1 7 . 8 1 5}$ & 2.423 \\
TRange & $\mathbf{9 . 9 9 0}$ & 1.692 & 5.610 \\
Soil Depth & 1.014 & 0.475 & $\mathbf{2 2 . 9 2 9}$ \\
pH & $\mathbf{1 1 . 6 4 2}$ & 1.065 & 1.072 \\
Clay & $\mathbf{7 . 5 1 5}$ & 7.186 & 0.206 \\
Silt & 0.158 & 12.643 & $\mathbf{1 8 . 9 3 4}$ \\
Sand & 4.071 & 1.731 & $\mathbf{1 6 . 9 6 1}$ \\
Soil Water & 7.881 & 0.506 & $\mathbf{1 1 . 4 2 2}$ \\
\hline
\end{tabular}

Table S3. Reference table for all the models fitted in this paper.

All models were implemented with and without accounting for the phylogeny. Refer to table S6 to see the whole model list. Variables to the right of the ${ }^{\sim}$ symbol are the independent variables, those to the left the dependent ones. VAR 1, models without fixed effects; ENV(1), environmental PCA fitted one by one; ENV, environmental PCA fitted all at once; Affiliation, Angiosperms/Gymnosperms grouping.

\begin{tabular}{|c|c|c|c|c|}
\hline Model & Description & Environment & Affiliation & Number of response varia \\
\hline $\operatorname{var}^{\sim} 1$ & Phylogenetic signal & No & No & Univariate \\
\hline trait $\sim \operatorname{env}(1)$ & Univariate trait models & Yes & No & Univariate \\
\hline trait $\sim$ env $(1)+$ Affiliation & & Yes & Yes & Univariate \\
\hline
\end{tabular}




\begin{tabular}{|c|c|c|c|c|}
\hline Model & Description & Environment & Affiliation & Number of response varic \\
\hline trait env $(1) *$ Affiliation & & Yes & Yes (interaction) & Univariate \\
\hline ht, ht -1 & Multi-response trait models & No & No & Bivariate \\
\hline ht, ht $\sim-1+$ Affiliation & & No & Yes & Bivariate \\
\hline ht, ht ${ }^{\sim}-1+$ env & & Yes & No & Bivariate \\
\hline ht, ht $\sim-1+$ env + Affiliation & & Yes & Yes & Bivariate \\
\hline ht, ht $\sim-1+$ env $*$ Affiliation & & Yes & Yes (interaction) & Bivariate \\
\hline
\end{tabular}

Table S4. Non-phylogenetic model's variance partition.

Mean and credible interval of the proportion of variance for non-phylogenetic inter-generic $(\gamma)$ and nonphylogenetic intra-generic $(\rho)$.

\begin{tabular}{llll}
\hline variable & Phylogenetic $(\lambda)$ & Inter-generic $(\gamma)$ & Intra-generic $(\rho)$ \\
\hline $\log (\mathrm{Hv})$ & 0 & $0.512(0.440,0.573)$ & $0.488(0.427,0.560)$ \\
$\log \left(K_{\mathrm{S}}\right)$ & 0 & $0.485(0.381,0.523)$ & $0.542(0.468,0.619)$ \\
$\log \left(\left|\psi_{\min }\right|\right)$ & 0 & $0.618(0.522,0.688)$ & $0.382(0.312,0.478)$ \\
$\log (|\mathrm{P} 50|)$ & 0 & $0.638(0.578,0.700)$ & $0.362(0.300,0.422)$ \\
$\mathrm{PC} 1$ & 0 & $0.768(0.742,0.796)$ & $0.232(0.204,0.258)$ \\
$\mathrm{PC} 2$ & 0 & $0.510(0.465,0.560)$ & $0.490(0.440,0.535)$ \\
PC3 & 0 & $0.627(0.588,0.668)$ & $0.373(0.332,0.412)$ \\
\hline
\end{tabular}

Table S5. Significant evolutionary correlations between pairs of variables without fixed effects (Fig. 3).

Mean (E. Correlation) and lower and upper 95\% credible intervals (HDP) are shown.

\begin{tabular}{lllll}
\hline $\operatorname{var} 1$ & var2 & E. Correlation & Lower HDP & Upper HDP \\
\hline $\log (\mathrm{Hv})$ & PC1 & -0.779 & -0.906 & -0.635 \\
$\log (\mathrm{Hv})$ & PC3 & 0.581 & 0.364 & 0.778 \\
$\log \left(K_{\mathrm{S}}\right)$ & $\log (\mathrm{Hv})$ & -0.607 & -0.882 & -0.277 \\
$\log \left(K_{\mathrm{s}}\right)$ & $\mathrm{PC} 1$ & 0.298 & 0.040 & 0.531 \\
$\log \left(K_{\mathrm{s}}\right)$ & PC3 & -0.443 & -0.694 & -0.185 \\
$\log \left(\left|\psi_{\min }\right|\right)$ & PC1 & -0.822 & -0.937 & -0.692 \\
$\log (|\mathrm{P} 50|)$ & $\log (\mathrm{Hv})$ & 0.414 & 0.024 & 0.772 \\
$\log (|\mathrm{P} 50|)$ & $\log \left(\left|\psi_{\min }\right|\right)$ & 0.693 & 0.412 & 0.905 \\
$\log (|\mathrm{P} 50|)$ & PC1 & -0.719 & -0.872 & -0.552 \\
\hline
\end{tabular}

Table S6. Univariate models description.

DICs and explained variances for phylogenetic and non-phylogenetic univariate models. Var_variance_partitioning: univariate model without fixed effects; Ht_Env1: univariate model with individual environmental principal components as fixed effects; DICs for the phylogenetic models are shown. NP refer to non_phylogenetic models (i.e., only including genus contingency as random effect) explained variances. R2c refer to the conditional and $\mathrm{R} 2 \mathrm{~m}$ refers to the marginal explained variances.

\begin{tabular}{|c|c|c|c|c|c|c|}
\hline name & fix.frml & DIC & $\mathrm{R} 2 \mathrm{~m}$ & $\mathrm{R} 2 \mathrm{c}$ & NP_R2m & NP_R2 \\
\hline Var_variance_patitioning_NG_HSM & $\mathrm{HSM} \sim 1$ & 2682.908 & 0 & 0.556 & 0 & 0.492 \\
\hline Ht_Env_1_GI_HSM_PC1 & HSM $\sim \mathrm{PC} 1 *$ Affiliation & 914.981 & 0.272 & 0.614 & 0.33 & 0.562 \\
\hline
\end{tabular}




\begin{tabular}{|c|c|c|c|c|c|c|}
\hline name & fix.frml & DIC & $\mathrm{R} 2 \mathrm{~m}$ & $\mathrm{R} 2 \mathrm{c}$ & NP_R2m & NP_R2 \\
\hline Ht_Env_1_NG_HSM_PC1 & $\mathrm{HSM} \sim \mathrm{PC} 1$ & 924.782 & 0.064 & 0.541 & 0.032 & 0.491 \\
\hline Ht_Env_1_G_HSM_PC1 & $\mathrm{HSM} \sim \mathrm{PC} 1+$ Affiliation & 926.332 & 0.222 & 0.578 & 0.279 & 0.507 \\
\hline Ht_Env_1_GI_HSM_PC2 & $\mathrm{HSM} \sim \mathrm{PC} 2 *$ Affiliation & 927.430 & 0.244 & 0.559 & 0.281 & 0.512 \\
\hline Ht_Env_1_NG_HSM_PC2 & $\mathrm{HSM} \sim \mathrm{PC} 2$ & 933.283 & 0.016 & 0.559 & 0.027 & 0.484 \\
\hline Ht_Env_1_G_HSM_PC2 & $\mathrm{HSM} \sim \mathrm{PC} 2+$ Affiliation & 933.751 & 0.182 & 0.609 & 0.233 & 0.528 \\
\hline Ht_Env_1_NG_HSM_PC3 & $\mathrm{HSM} \sim \mathrm{PC} 3$ & 929.952 & 0.028 & 0.55 & 0.042 & 0.507 \\
\hline Ht_Env_1_G_HSM_PC3 & HSM $\sim \mathrm{PC} 3+$ Affiliation & 930.497 & 0.19 & 0.585 & 0.244 & 0.526 \\
\hline Ht_Env_1_GI_HSM_PC3 & HSM PC3 $*$ Affiliation & 931.240 & 0.194 & 0.599 & 0.249 & 0.528 \\
\hline Var_variance_patitioning_NG_log(Hv) & $\log (\mathrm{Hv}) \sim 1$ & 3148.379 & 0 & 0.637 & 0 & 0.512 \\
\hline Ht_Env_1_G_log(Hv)_PC1 & $\log (\mathrm{Hv}) \sim \mathrm{PC} 1+$ Affiliation & 3067.336 & 0.147 & 0.563 & 0.166 & 0.488 \\
\hline Ht_Env_1_NG_log(Hv)_PC1 & $\log (\mathrm{Hv}) \sim \mathrm{PC} 1$ & 3067.384 & 0.134 & 0.55 & 0.164 & 0.485 \\
\hline Ht_Env_1_GI_log(Hv)_PC1 & $\log (\mathrm{Hv}) \sim \mathrm{PC} 1 *$ Affiliation & 3069.835 & 0.148 & 0.567 & 0.167 & 0.487 \\
\hline Ht_Env_1_GI_log(Hv)_PC2 & $\log (\mathrm{Hv}) \sim \mathrm{PC} 2 *$ Affiliation & 684.776 & 0.029 & 0.665 & 0.013 & 0.516 \\
\hline Ht_Env_1_G_log(Hv)_PC2 & $\log (\mathrm{Hv}) \sim \mathrm{PC} 2+$ Affiliation & 702.888 & 0.028 & 0.663 & 0.012 & 0.514 \\
\hline Ht_Env_1_NG_log(Hv)_PC2 & $\log (\mathrm{Hv}) \sim \mathrm{PC} 2$ & 702.977 & 0.001 & 0.648 & 0.001 & 0.516 \\
\hline Ht_Env_1_G_log(Hv)_PC3 & $\log (\mathrm{Hv}) \sim \mathrm{PC} 3+$ Affiliation & 738.895 & 0.052 & 0.62 & 0.05 & 0.489 \\
\hline Ht_Env_1_NG_log(Hv)_PC3 & $\log (\mathrm{Hv}) \sim \mathrm{PC} 3$ & 738.990 & 0.029 & 0.606 & 0.044 & 0.49 \\
\hline Ht_Env_1_GI_log(Hv)_PC3 & $\log (\mathrm{Hv}) \sim \mathrm{PC} 3 *$ Affiliation & 739.002 & 0.052 & 0.619 & 0.053 & 0.486 \\
\hline Var_variance_patitioning_NG_log(Kl) & $\log (\mathrm{Kl}) \sim 1$ & 2706.985 & 0 & 0.468 & 0 & 0.28 \\
\hline Ht_Env_1_GI_log(Kl)_PC1 & $\log (\mathrm{Kl}) \sim \mathrm{PC} 1 *$ Affiliation & 2276.884 & 0.065 & 0.489 & 0.077 & 0.295 \\
\hline Ht_Env_1_NG_log(Kl)_PC1 & $\log (\mathrm{Kl}) \sim \mathrm{PC} 1$ & 2282.091 & 0.001 & 0.448 & 0.002 & 0.274 \\
\hline Ht_Env_1_G_log(Kl)_PC1 & $\log (\mathrm{Kl}) \sim \mathrm{PC} 1+$ Affiliation & 2283.858 & 0.067 & 0.465 & 0.073 & 0.285 \\
\hline Ht_Env_1_NG_log(Kl)_PC2 & $\log (\mathrm{Kl}) \sim \mathrm{PC} 2$ & 2279.126 & 0.016 & 0.412 & 0.03 & 0.245 \\
\hline Ht_Env_1_GI_log(Kl)_PC2 & $\log (\mathrm{Kl}) \sim \mathrm{PC} 2 *$ Affiliation & 2279.593 & 0.082 & 0.449 & 0.09 & 0.272 \\
\hline Ht_Env_1_G_log(Kl)_PC2 & $\log (\mathrm{Kl}) \sim \mathrm{PC} 2+$ Affiliation & 2280.022 & 0.08 & 0.445 & 0.086 & 0.269 \\
\hline Ht_Env_1_NG_log(Kl)_PC3 & $\log (\mathrm{Kl}) \sim \mathrm{PC} 3$ & 2281.577 & 0.001 & 0.449 & 0.007 & 0.286 \\
\hline Ht_Env_1_GI_log(Kl)_PC3 & $\log (\mathrm{Kl}) \sim \mathrm{PC} 3 *$ Affiliation & 2282.851 & 0.07 & 0.468 & 0.074 & 0.289 \\
\hline Ht_Env_1_G_log(Kl)_PC3 & $\log (\mathrm{Kl}) \sim \mathrm{PC} 3+$ Affiliation & 2283.060 & 0.067 & 0.472 & 0.073 & 0.287 \\
\hline Var_variance_patitioning_NG_log(Ks) & $\log (\mathrm{Ks}) \sim 1$ & 2790.486 & 0 & 0.601 & 0 & 0.458 \\
\hline Ht_Env_1_NG_log(Ks)_PC1 & $\log (\mathrm{Ks}) \sim \mathrm{PC} 1$ & 2693.064 & 0.024 & 0.616 & 0.038 & 0.481 \\
\hline Ht_Env_1_G_log(Ks)_PC1 & $\log (\mathrm{Ks}) \sim \mathrm{PC} 1+$ Affiliation & 2693.094 & 0.08 & 0.637 & 0.084 & 0.483 \\
\hline Ht_Env_1_GI_log(Ks)_PC1 & $\log (\mathrm{Ks}) \sim \mathrm{PC} 1 *$ Affiliation & 2694.553 & 0.088 & 0.645 & 0.086 & 0.486 \\
\hline Ht_Env_1_NG_log(Ks)_PC2 & $\log (\mathrm{Ks}) \sim \mathrm{PC} 2$ & 1410.836 & 0.004 & 0.609 & 0.011 & 0.456 \\
\hline Ht_Env_1_G_log(Ks)_PC2 & $\log (\mathrm{Ks}) \sim \mathrm{PC} 2+$ Affiliation & 1411.862 & 0.06 & 0.632 & 0.055 & 0.462 \\
\hline Ht_Env_1_GI_log(Ks)_PC2 & $\log (\mathrm{Ks}) \sim \mathrm{PC} 2 *$ Affiliation & 1413.381 & 0.056 & 0.634 & 0.056 & 0.461 \\
\hline Ht_Env_1_GI_log(Ks)_PC3 & $\log (\mathrm{Ks}) \sim \mathrm{PC} 3 *$ Affiliation & 1398.159 & 0.068 & 0.626 & 0.074 & 0.473 \\
\hline Ht_Env_1_G_log(Ks)_PC3 & $\log (\mathrm{Ks}) \sim \mathrm{PC} 3+$ Affiliation & 1402.383 & 0.067 & 0.623 & 0.074 & 0.473 \\
\hline Ht_Env_1_NG_log(Ks)_PC3 & $\log (\mathrm{Ks}) \sim \mathrm{PC} 3$ & 1402.451 & 0.015 & 0.597 & 0.037 & 0.469 \\
\hline Var_variance_patitioning_NG_log $(|\psi \min |)$ & $\log (|\psi \min |)^{\sim} 1$ & 837.435 & 0 & 0.811 & 0 & 0.618 \\
\hline Ht_Env_1_G_log $(|\psi \min |)$ PC1 & $\log (|\psi \min |) \sim \mathrm{PC} 1+$ Affiliation & 804.466 & 0.24 & 0.763 & 0.312 & 0.669 \\
\hline Ht_Env_1_NG_log $(|\psi \min |)$ _PC1 & $\log (|\psi \min |)^{\sim} \mathrm{PC} 1$ & 804.595 & 0.225 & 0.745 & 0.311 & 0.665 \\
\hline 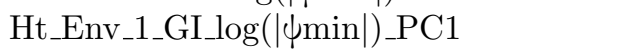 & $\log (|\psi \min |)^{\sim} \mathrm{PC} 1 *$ Affiliation & 806.659 & 0.243 & 0.783 & 0.318 & 0.69 \\
\hline Ht_Env_1_GI_log( $(|\psi \min |)$ _PC2 & $\log (|\psi \min |) \sim \mathrm{PC} 2 *$ Affiliation & 940.249 & 0.092 & 0.858 & 0.097 & 0.689 \\
\hline 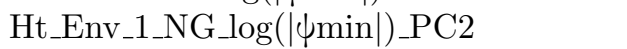 & $\log (|\psi \min |) \sim \mathrm{PC} 2$ & 2683.206 & 0.048 & 0.849 & 0.085 & 0.691 \\
\hline 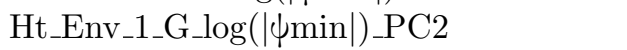 & $\log (|\psi \min |) \sim \mathrm{PC} 2+$ Affiliation & 2683.400 & 0.087 & 0.858 & 0.093 & 0.687 \\
\hline Ht_Env_1_GI_log $(|\psi \min |)$ PC3 & $\log (|\psi \min |) \sim \mathrm{PC} 3 *$ Affiliation & 2342.085 & 0.069 & 0.834 & 0.053 & 0.639 \\
\hline 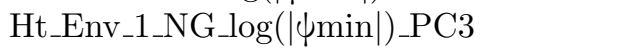 & $\log (|\psi \min |) \sim \mathrm{PC} 3$ & 2707.060 & 0.018 & 0.822 & 0.038 & 0.635 \\
\hline 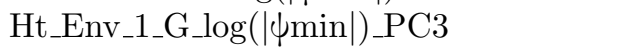 & $\log (|\psi \min |) \sim \mathrm{PC} 3+$ Affiliation & 2709.044 & 0.072 & 0.835 & 0.052 & 0.641 \\
\hline Var_variance_patitioning_NG_log(|P50|) & $\log (|\mathrm{P} 50|) \sim 1$ & 1426.768 & 0 & 0.709 & 0 & 0.638 \\
\hline Ht_Env_1_G_log(|P50|)_PC1 & $\log (|\mathrm{P} 50|) \sim \mathrm{PC} 1+$ Affiliation & 1402.439 & 0.166 & 0.635 & 0.204 & 0.594 \\
\hline
\end{tabular}




\begin{tabular}{lllllll}
\hline name & fix.frml & DIC & R2m & R2c & NP_R2m & NP_R2c \\
\hline Ht_Env_1_NG_log(|P50|)_PC1 & $\log (|\mathrm{P} 50|) \sim$ PC1 & 1402.584 & 0.04 & 0.62 & 0.065 & 0.577 \\
Ht_Env_1_GI_log(|P50|)_PC1 & $\log (|\mathrm{P} 50|) \sim$ PC1 * Affiliation & 1404.263 & 0.164 & 0.633 & 0.203 & 0.596 \\
Ht_Env_1_G_log(|P50|)_PC2 & $\log (|\mathrm{P} 50|) \sim$ PC2 + Affiliation & 3026.572 & 0.106 & 0.72 & 0.137 & 0.628 \\
Ht_Env_1_NG_log(|P50|)_PC2 & $\log (|\mathrm{P} 50|) \sim$ PC2 & 3026.704 & 0.001 & 0.693 & 0.005 & 0.62 \\
Ht_Env_1_GI_log(|P50|)_PC2 & $\log (|\mathrm{P} 50|) \sim$ PC2 * Affiliation & 3026.963 & 0.113 & 0.719 & 0.147 & 0.633 \\
Ht_Env_1_NG_log(|P50|)_PC3 & $\log (|\mathrm{P} 50|) \sim$ PC3 & 3071.094 & 0.001 & 0.693 & 0.002 & 0.617 \\
Ht_Env_1_G_log(|P50|)_PC3 & $\log (|\mathrm{P} 50|) \sim$ PC3 + Affiliation & 3071.125 & 0.108 & 0.72 & 0.139 & 0.627 \\
Ht_Env_1_GI_log(|P50|)_PC3 & $\log (|\mathrm{P} 50|) \sim$ PC3 * Affiliation & 3071.472 & 0.107 & 0.722 & 0.142 & 0.628 \\
Var_variance_patitioning_NG_PC1 & PC1 $\sim 1$ & 6980.484 & 0 & 0.883 & 0 & 0.768 \\
Var_variance_patitioning_NG_PC2 & PC2 $\sim 1$ & 7089.458 & 0 & 0.714 & 0 & 0.51 \\
Var_variance_patitioning_NG_PC3 & PC3 $\sim 1$ & 5678.017 & 0 & 0.848 & 0 & 0.627 \\
\hline
\end{tabular}

Table S7. Evolutionary correlations and DICs for multivariate models.

Models are ordered by DIC values (from lower to higher) for each set of nested models (same response variables). Significant correlations are highlighted in bold.

\begin{tabular}{|c|c|c|}
\hline var1 & var2 & fix.frml \\
\hline $\log (\mathrm{Hv})$ & $\log (|\psi \min |)$ & $\operatorname{cbind}\left(\log (\mathrm{Hv}) \_\log (|\psi \min |)\right)^{\sim}$ trait-1 + (trait:PC1 + trait:PC2 + trait:PC3) * trait:Affiliat \\
\hline $\log (\mathrm{Hv})$ & $\log (|\psi \min |)$ & $\operatorname{cbind}(\log (\mathrm{Hv}) \log (|\psi \min |))$ trait-1 + trait:PC1 + trait:PC2 + trait:PC3 \\
\hline $\log (\mathrm{Hv})$ & $\log (|\psi \min |)$ & $\operatorname{cbind}\left(\log \left(\mathrm{Hv}_{\mathrm{v}} \log _{(}(|\psi \min |)\right) \sim\right.$ trait-1 + trait:Affiliation + trait:PC1 + trait:PC2 + trait:PC \\
\hline $\log (\mathrm{Hv})$ & $\log (|\psi \min |)$ & $\operatorname{cbind}(\log (\mathrm{Hv}) \log (|\psi \min |)) \sim$ trait-1 + trait:Affiliation \\
\hline $\log (\mathrm{Hv})$ & $\log (|\psi \min |)$ & $\operatorname{cbind}\left(\log \left(\mathrm{Hv}_{\mathrm{v}} \log _{(}(|\psi \min |)\right) \sim\right.$ trait-1 \\
\hline $\log (\mathrm{Hv})$ & PC1 & $\operatorname{cbind}\left(\log (\mathrm{Hv}) \_\mathbf{P C} 1\right) \sim$ trait-1 \\
\hline $\log (\mathrm{Hv})$ & PC1 & cbind $\left(\log (\mathbf{H v}) \_P C 1\right) \sim$ trait-1 + trait:Affiliation \\
\hline $\log (\mathrm{Hv})$ & $\mathrm{PC} 2$ & $\operatorname{cbind}\left(\log (\mathrm{Hv}) \_\mathrm{PC} 2\right) \sim$ trait-1 + trait:Affiliation \\
\hline $\log (\mathrm{Hv})$ & $\mathrm{PC} 2$ & cbind $\left(\log (\mathrm{Hv}) \_\mathrm{PC} 2\right) \sim$ trait-1 \\
\hline $\log (\mathbf{H v})$ & PC3 & $\operatorname{cbind}(\log (\mathbf{H v})$ PC3 $) \sim$ trait-1 + trait:Affiliation \\
\hline $\log (\mathrm{Hv})$ & PC3 & $\operatorname{cbind}\left(\log (\mathrm{Hv}) \_\right.$PC3 $)$ trait-1 \\
\hline $\log (\mathrm{Ks})$ & $\log (\mathbf{H v})$ & cbind $\left(\log (\mathrm{Ks}) \_\log (\mathbf{H v})\right) \sim$ trait-1 + trait:PC1 + trait:PC2 + trait:PC3 \\
\hline $\log (\mathrm{Ks})$ & $\log (\mathrm{Hv})$ & $\operatorname{cbind}(\log (\mathrm{Ks}) \log (\mathrm{Hv})) \sim$ trait-1 + trait:Affiliation + trait:PC1 + trait:PC2 \\
\hline $\log (\mathrm{Ks})$ & $\log (\mathrm{Hv})$ & $\operatorname{cbind}(\log (\mathrm{Ks}) \log (\mathrm{Hv})) \sim$ trait-1 $+($ trait:PC1 + trait:PC2 + trait:PC3 $) *$ tra \\
\hline $\log (\mathrm{Ks})$ & $\log (\mathrm{Hv})$ & $\operatorname{cbind}\left(\log (\mathrm{Ks}) \_l o g(\mathrm{Hv})\right) \sim$ trait-1 \\
\hline $\log (\mathrm{Ks})$ & $\log (\mathrm{Hv})$ & $\operatorname{cbind}(\log (\mathbf{K s}) \log (\mathbf{H v})) \sim$ trait-1 + trait:Affiliation \\
\hline $\log (\mathrm{Ks})$ & $\log (|\psi \min |)$ & $\operatorname{cbind}(\log (\mathrm{Ks}) \log (|\psi \min |))^{\sim}$ trait-1 + (trait:PC1 + trait:PC2 + trait:PC3) * trait:Affiliati \\
\hline $\log (\mathrm{Ks})$ & $\log (|\psi \min |)$ & $\operatorname{cbind}(\log (\mathrm{Ks}) \log (|\psi \min |)) \sim$ trait-1 + trait:Affiliation + trait:PC1 + trait:PC2 + trait:PC \\
\hline $\log (\mathrm{Ks})$ & $\log (|\psi \min |)$ & cbind $(\log (\mathrm{Ks}) \log (|\psi \min |)) \sim$ trait-1 + trait:PC1 + trait:PC2 + trait:PC3 \\
\hline $\log (\mathrm{Ks})$ & $\log (|\psi \min |)$ & $\operatorname{cbind}\left(\log (\mathrm{Ks}) \_\log (|\psi \min |)\right) \sim$ trait-1 \\
\hline $\log (\mathrm{Ks})$ & $\log (|\psi \min |)$ & $\operatorname{cbind}(\log (\mathrm{Ks}) \log (|\psi \min |)) \sim$ trait-1 + trait:Affiliation \\
\hline $\log (\mathrm{Ks})$ & $\log (|\mathrm{P} 50|)$ & cbind $\left(\log (\mathrm{Ks}) \_\log (|\mathrm{P} 50|)\right) \sim$ trait-1 + trait:Affiliation + trait:PC1 + trait:PC2 + trait:PC \\
\hline $\log (\mathrm{Ks})$ & $\log (|\mathrm{P} 50|)$ & $\operatorname{cbind}(\log (\mathrm{Ks}) \log (|\mathrm{P} 50|)) \sim$ trait-1 + trait:PC1 + trait:PC2 + trait:PC3 \\
\hline $\log (\mathrm{Ks})$ & $\log (|\mathrm{P} 50|)$ & $\operatorname{cbind}\left(\log (\mathrm{Ks}) \_\log (|\mathrm{P} 50|)\right) \sim$ trait-1 $+($ trait:PC1 + trait:PC2 + trait:PC3) * trait:Affiliatic \\
\hline $\log (\mathrm{Ks})$ & $\log (|\mathrm{P} 50|)$ & $\operatorname{cbind}(\log (\mathrm{Ks}) \log (|\mathrm{P} 50|)) \sim$ trait-1 \\
\hline $\log (\mathrm{Ks})$ & $\log (|\mathrm{P} 50|)$ & $\operatorname{cbind}\left(\log (\mathrm{Ks}) \_\log (|\mathrm{P} 50|)\right)$ trait-1 + trait:Affiliation \\
\hline $\log (\mathrm{Ks})$ & PC1 & $\operatorname{cbind}\left(\log (\mathrm{Ks}) \_\mathbf{P C} 1\right) \sim$ trait-1 + trait:Affiliation \\
\hline $\log (\mathbf{K s})$ & PC1 & $\operatorname{cbind}\left(\log (\mathrm{Ks}) \_\mathrm{PC} 1\right) \sim$ trait-1 \\
\hline $\log (\mathrm{Ks})$ & $\mathrm{PC} 2$ & $\operatorname{cbind}\left(\log (\mathrm{Ks}) \_\mathrm{PC} 2\right) \sim$ trait-1 + trait:Affiliation \\
\hline $\log (\mathrm{Ks})$ & $\mathrm{PC} 2$ & $\operatorname{cbind}\left(\log (\mathrm{Ks}) \_\mathrm{PC} 2\right) \sim$ trait-1 \\
\hline $\log (\mathrm{Ks})$ & PC3 & $\operatorname{cbind}\left(\log (\mathrm{Ks}) \_\mathbf{P C} 3\right) \sim$ trait-1 + trait:Affiliation \\
\hline
\end{tabular}




\begin{tabular}{|c|c|c|}
\hline var1 & var2 & fix.frml \\
\hline $\log (\mathbf{K s})$ & PC3 & $\operatorname{cbind}\left(\log (\mathrm{Ks}) \_\mathrm{PC} 3\right) \sim$ trait-1 \\
\hline$\lambda \operatorname{o\gamma }(|\psi \mu \omega \nu|)$ & PC1 & $\varsigma \beta \iota \nu \delta\left(\lambda \circ \gamma(|\psi \mu \iota \nu|) \_\Pi " 1\right) \sim \tau \rho \alpha \iota \tau-1+\tau \rho \alpha \iota \tau: \mathrm{A} \varphi \varphi \iota \lambda \iota \alpha \tau \iota \nu \nu$ \\
\hline$\lambda \operatorname{o\gamma }(|\psi \mu \nu \nu|)$ & PC1 & $\varsigma \beta \iota \nu \delta\left(\lambda o \gamma(|\psi \mu \nu \nu|)+\Pi^{\prime \prime} 1\right) \sim \tau \rho \alpha \tau-1$ \\
\hline $\log (|\psi \min |)$ & $\mathrm{PC} 2$ & cbind $\left(\log (|\psi \min |) \_\right.$PC2 $)$ trait-1 \\
\hline $\log (|\psi \min |)$ & $\mathrm{PC} 2$ & $\operatorname{cbind}(\log (|\psi \min |)+\mathrm{PC} 2) \sim$ trait-1 + trait:Affiliation \\
\hline $\log (|\psi \min |)$ & PC3 & $\operatorname{cbind}\left(\log (|\psi \min |) \_\right.$PC3 $) \sim$ trait-1 + trait:Affiliation \\
\hline $\log (|\psi \min |)$ & PC3 & $\operatorname{cbind}(\log (|\psi \min |)+\mathrm{PC} 3) \sim$ trait-1 \\
\hline $\log (|\mathrm{P} 50|)$ & $\log (\mathrm{Hv})$ & cbind $(\log (|\mathrm{P} 50|) \log (\mathrm{Hv})) \sim$ trait-1 + trait:PC1 + trait:PC2 + trait:PC3 \\
\hline $\log (|\mathrm{P} 50|)$ & $\log (\mathrm{Hv})$ & $\operatorname{cbind}(\log (|\mathrm{P} 50|) \log (\mathrm{Hv})) \sim$ trait-1 + trait:Affiliation + trait:PC1 + trait:PC2 + trait:PC3 \\
\hline $\log (|\mathrm{P} 50|)$ & $\log (\mathrm{Hv})$ & $\operatorname{cbind}(\log (|\mathrm{P} 50|) \log (\mathrm{Hv})) \sim$ trait-1 $+($ trait:PC $1+$ trait:PC $2+$ trait:PC3 $) *$ trait:Affiliatio \\
\hline $\log (|\mathbf{P} 50|)$ & $\log (\mathbf{H v})$ & $\operatorname{cbind}\left(\log (|\mathrm{P} 50|) \_\log (\mathbf{H v})\right) \sim$ trait-1 \\
\hline $\log (|\mathrm{P} 50|)$ & $\log (\mathrm{Hv})$ & $\operatorname{cbind}\left(\log (|\mathrm{P} 50|) \_\log (\mathrm{Hv})\right) \sim$ trait-1 + trait:Affiliation \\
\hline $\log (|\mathbf{P} 50|)$ & $\lambda o \gamma(|\psi \mu \omega|)$ & $\varsigma \beta \iota \nu \delta\left(\lambda \circ \gamma(|\Pi 50|) \_\lambda \circ \gamma(|\psi \mu \iota \nu|)^{\sim} \tau \rho \alpha \iota \tau-1+\left(\tau \rho \alpha l \tau: \Pi \Pi^{\prime \prime} 1+\tau \rho \alpha \iota \tau: \Pi^{\prime \prime} 2+\tau \rho \alpha \iota \tau\right.\right.$ \\
\hline $\log (|\mathbf{P} 50|)$ & $\lambda o \gamma(|\psi \mu \nu|)$ & $\varsigma \beta \iota \nu \delta\left(\lambda \circ \gamma(|\Pi 50|) \lambda \operatorname{lo\gamma }(|\psi \mu \iota \nu|)^{\sim} \tau \rho \alpha \iota \tau-1+\tau \rho \alpha \iota \tau: \mathrm{A} \varphi \varphi \iota \lambda \iota \alpha \tau \iota 0 \nu+\tau p \alpha \iota \tau: \Pi^{\prime \prime} 1\right.$ \\
\hline $\log (|\mathbf{P} 50|)$ & $\lambda o \gamma(|\psi \mu \nu|)$ & $\varsigma \beta \iota \nu \delta\left(\lambda \circ \gamma(|\Pi 50|) \_\lambda \circ \gamma(|\psi \mu \iota \nu|)^{\sim} \tau \rho \alpha \iota \tau-1+\tau \rho \alpha \iota \tau: \Pi^{\prime \prime} 1+\tau \rho \alpha \iota \tau: \Pi^{\prime \prime} 2+\tau \rho \alpha \iota \tau:\right.$ \\
\hline $\log (|\mathbf{P} 50|)$ & $\lambda o \gamma(|\psi \mu \nu|)$ & $\varsigma \beta \iota \nu \delta\left(\lambda \circ \gamma(|\Pi 50|) \_\lambda \circ \gamma(|\psi \mu \iota \nu| » \sim \tau \rho \alpha \iota \tau-1+\tau \rho \alpha \iota \tau: \mathrm{A} \varphi \varphi \iota \lambda \iota \alpha \tau \iota 0 \nu\right.$ \\
\hline $\log (|\mathbf{P} 50|)$ & $\lambda o \gamma(|\psi \mu \omega \nu|)$ & $\varsigma \beta \iota \nu \delta\left(\lambda \circ \gamma(|\Pi 50|) \_\lambda o \gamma(|\psi \mu \nu \nu| »)^{\sim} \tau \rho \iota \tau-1\right.$ \\
\hline $\log (|\mathbf{P} 50|)$ & PC1 & $\operatorname{cbind}\left(\log (|\mathrm{P} 50|) \_\mathrm{PC} 1\right) \sim$ trait-1 + trait:Affiliation \\
\hline $\log (|\mathbf{P} 50|)$ & PC1 & $\operatorname{cbind}\left(\log (|\mathrm{P} 50|) \_\mathrm{PC} 1\right) \sim$ trait-1 \\
\hline $\log (|\mathrm{P} 50|)$ & $\mathrm{PC} 2$ & $\operatorname{cbind}\left(\log (|\mathrm{P} 50|) \_\mathrm{PC} 2\right) \sim$ trait-1 \\
\hline $\log (|\mathrm{P} 50|)$ & $\mathrm{PC} 2$ & $\operatorname{cbind}\left(\log (|\mathrm{P} 50|) \_\mathrm{PC} 2\right) \sim$ trait-1 + trait:Affiliation \\
\hline $\log (|\mathrm{P} 50|)$ & PC3 & $\operatorname{cbind}(\log (|\mathrm{P} 50|)$ PC3 $) \sim$ trait-1 + trait:Affiliation \\
\hline $\log (|\mathrm{P} 50|)$ & PC3 & $\operatorname{cbind}\left(\log (|\mathrm{P} 50|) \_\mathrm{PC} 3\right)^{\sim}$ trait-1 \\
\hline
\end{tabular}

Appendix S1. Supplementary methods.

Phylogenetic mixed model description

Phylogenetic mixed models are commonly used in quantitative genetics (the so called "animal" model), being useful for comparative analyses as they allow to incorporate a range of variance structures for the random effects, including shared ancestry through a phylogeny (Housworthet al. 2004). The general model structure is defined as follows:

$y=\mu+\beta \xi+p+g+e(1)$

Were $\mu$ is the grand mean, interpreted as the root ancestor state, $\beta$ is the slope for the covariate $\mathrm{x}$ (fixed effect, in green), $p$ and $g$ are the variability caused by the genus-level phylogeny and the genus contingency effects (random effects, in red), and $e$ is the residual error (Housworth et al. 2004; Villemereuil \& Nakagawa 2014). Both fixed $(\beta)$ and random ( $r$, which is $p+g$ ) effects and the residuals (e) are expected to come from a multivariate normal distribution as it follows:

$$
\begin{aligned}
& {\left[\begin{array}{l}
\beta \\
r \\
e
\end{array}\right] \sim N(} \\
& {\left[\begin{array}{l}
0 \\
0
\end{array}\right]} \\
& {\left[\begin{array}{lrl}
B & a m p ; 0 & a m p ; 0 \\
0 & a m p ; & G \\
0 & a m p ; & 0 \\
0
\end{array}\right]}
\end{aligned}
$$


Where $\beta$ is the fixed effect parameter to estimate, $\beta_{0}$ are the prior means for the fixed effects with prior (co)variance matrix $\mathrm{B}$, and $\mathrm{G}$ and $\mathrm{R}$ are the expected (co)variances of the random effects and the residuals respectively (Hadfield 2010; Hadfield \& Nakagawa 2010). G and R are unknown, and must be estimated from the data by assuming they are structured in a way that can be parametrized by few parameters, as it has been exemplified below for the $\mathrm{G}$ case:

$G=$

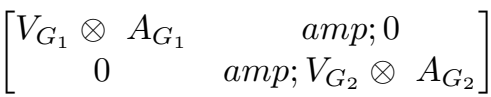

Were the (co)variance matrices $(\mathrm{V})$ are matrices with one parameter to be estimated per response variable and the structured matrices $(A)$ refer to the phylogenetic structure $\left(\mathrm{A}_{\mathrm{G} 1}\right)$ and genus contingency $\left(\mathrm{A}_{\mathrm{G} 2}\right)$. The Kronecker product ([?]) allows for possible dependence between random effects (Hadfield 2010; Hadfield \& Nakagawa 2010).

In multi-response models, the (co)variance matrix of the previous equation is reformulated including the covariance estimates in the off-diagonal and the respective variances in the diagonal as follows:

$V_{G 1}=$

] (4)

Where $\sigma^{2}{ }_{\mathrm{u} 1}$ is the variance for the first response variable $\left(\mathrm{V}_{1}\right)$ and $\sigma^{2}{ }_{\mathrm{u} 2}$ the variance for the second response variable $\left(\mathrm{V}_{2}\right)$, while $\sigma_{\mathrm{u} 1, \mathrm{u} 2}$ and $\sigma_{\mathrm{u} 2, \mathrm{u} 1}$ are the same covariance estimate $(\mathrm{C})$.

Phylogenetic indexes calculation

The phylogenetic signal or phylogenetic heritability it is calculated as follows ( Villemereuil \& Nakagawa 2014):

$\lambda=\frac{\sigma_{p}^{2}}{\sigma_{p}^{2}+\sigma_{g}^{2}+\sigma_{e}^{2}}(5)$

Where $\sigma_{P}^{2}$ is the variance of the phylogenetic effect $\left(\mathrm{V}_{\mathrm{G} 1}\right), \sigma_{g}^{2}$ is the variance of the cross-genus effect $\left(\mathrm{V}_{\mathrm{G} 2}\right)$ and $\sigma_{e}^{2}$ is the residual error (Villemereuil \& Nakagawa 2014). Cross-genera variance (i.e. non-phylogenetic variation among genera or genus lability) has been calculated as follows:

$\gamma=\frac{\sigma_{g}^{2}}{\sigma_{p}^{2}+\sigma_{g}^{2}+\sigma_{e}^{2}}(6)$

And finally, intra-genus variability including measurement error has been calculated as follows:

$\rho=\frac{\sigma_{e}^{2}}{\sigma_{p}^{2}+\sigma_{g}^{2}+\sigma_{e}^{2}}(7)$

Note also that $\gamma+\rho+\lambda=1$ (Housworth et al. 2004). The three indexes were calculated for the whole Markov chain random effects and residual samples (once burned and thinned), so the output is a statistical distribution from which the mean and $95 \%$ credible intervals can be calculated.

\section{Phylogenetic covariation calculation}

From the phylogenetic variances and covariance obtained in equation 4, the evolutionary correlation between response variables can be calculated as follows (Villemereuil 2012):

$r_{\mathrm{ev}}=\frac{\sigma_{u_{2}, u_{1}}}{\sqrt{\sigma_{u_{1}}^{2} \sigma_{u_{2}}^{2}}}(8)$

\section{Model specifications}

Univariate models random effects variance priors were set as $\mathrm{V}=1, \mathrm{nu}=0.002$. For bivariate models, the random effects variances priors were set as $\mathrm{V}=\operatorname{diag}(2) / 2, \mathrm{nu}=2$. To achieve convergence, each model was run for 8,000,000 iterations with a 1,000,000 burn-in and a thinning interval of 4,000, reaching an effective sample size between 1,000 and 2,000 in all estimated parameters. When models did not converge, 
we increased the number of iterations until convergence were achieved. Thinning intervals and the final number of iterations were progressively increased until autocorrelations between samples were found to be $<0.1$. Convergence of all models was assessed by plots of chain mixing and by the Heidenberg stationary test as a diagnostic. All reported models had a low degree of autocorrelation between iterations and passed the convergence diagnostic, both for fixed and random effects (i.e., the sampled chains were stationary).

\section{Literature cited}

Hadfield, J.D. (2010). MCMCglmm for R. J. Stat. Softw. , 33.

Hadfield, J.D. \& Nakagawa, S. (2010). General quantitative genetic methods for comparative biology: Phylogenies, taxonomies and multi-trait models for continuous and categorical characters. J. Evol. Biol. , $23,494-508$.

Housworth, E.A., Martins, E.P. \& Lynch, M. (2004). The Phylogenetic Mixed Model. Am. Nat. , 163, 84-96.

Villemereuil, P. (2012). How to use the MCMCglmm R package.

Villemereuil, P. \& Nakagawa, S. (2014). General Quantitative Genetic Methods for Comparative Biology. In: (Modern Phylogenetic Comparative Methods and Their Application in Evolutionary Biology: Concepts and Practice ), \{[Garamszegi, L.Z.]\}. Springer, New York, USA. 287-303.

Appendix S2. Species-level phylogenetic analyses.

Species-level phylogeny was obtained by pruning the phylogenetic tree reported by Smith \& Brown (2018) available in the R package "v.PhyloMaker" (Jin \& Qian 2019) by using the "ape" R package (Paradis \& Schliep 2018) only keeping species with hydraulic data available in each case, obtaining the same number of observations compared to the genus-level analyses. All bivariate models implemented using the genus-level phylogeny where also conducted using the species-level phylogeny. As we had only one value per specie, no extra random effect was included, so variance partition was reduced to phylogenetic signal calculation.

Phylogenetic signal results:

\begin{tabular}{llll}
\hline variable & lambdaMean & lambda_lowerHPD & lambda_upperHPD \\
\hline log_Hv & 0.670 & 0.583 & 0.749 \\
log_Ks & 0.636 & 0.533 & 0.723 \\
log_negMinWP_md & 0.818 & 0.761 & 0.876 \\
log_negP50 & 0.736 & 0.653 & 0.808 \\
PC1 & 0.908 & 0.887 & 0.926 \\
PC2 & 0.685 & 0.625 & 0.750 \\
PC3 & 0.858 & 0.827 & 0.886 \\
\hline
\end{tabular}

Evolutionary correlation results, those differing from the genus-level are highlighted in bold.

\begin{tabular}{|c|c|c|c|}
\hline model & Fixed formula & $\operatorname{var} 1$ & var2 \\
\hline 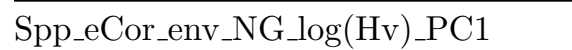 & $($ Env, trait $) \sim 1$ & $\log (\mathrm{Hv})$ & $\mathrm{PC} 1$ \\
\hline Spp_eCor_env_NG_log(Hv)_PC2 & $($ Env, trait $) \sim 1$ & $\log (\mathrm{Hv})$ & $\mathrm{PC} 2$ \\
\hline Spp_eCor_env_NG_log(Hv)_PC3 & (Env, trait) $\sim 1$ & $\log (\mathrm{Hv})$ & PC3 \\
\hline Spp_eCor_env_NG_log(Ks)_PC1 & (Env, trait) 1 & $\log (\mathrm{Ks})$ & $\mathrm{PC} 1$ \\
\hline Spp_eCor_env_NG_log(Ks)_PC2 & $($ Env, trait $) \sim 1$ & $\log (\mathrm{Ks})$ & PC2 \\
\hline Spp_eCor_env_NG_log(Ks)_PC3 & $($ Env, trait) $\sim 1$ & $\log (\mathrm{Ks})$ & $\mathrm{PC} 3$ \\
\hline 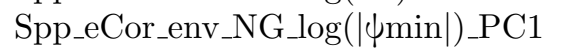 & $($ Env, trait $) \sim 1$ & $\log (|\psi \min |)$ & $\mathrm{PC} 1$ \\
\hline 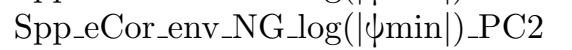 & (Env, trait) $\sim 1$ & $\log (|\psi \min |)$ & $\mathrm{PC} 2$ \\
\hline
\end{tabular}




\begin{tabular}{|c|c|c|c|}
\hline model & Fixed formula & $\operatorname{var} 1$ & var2 \\
\hline 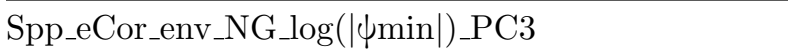 & $($ Env, trait $) \sim 1$ & $\log (|\psi \min |)$ & $\mathrm{PC} 3$ \\
\hline ppp_eCor_env_NG_log(|P50|)_PC1 & Env, trait) 1 & $\log (|\mathrm{P} 50|)$ & PC1 \\
\hline ppp_eCor_env_NG_log(|P50|)_PC2 & $($ Env, trait $) \sim 1$ & $\log (|\mathrm{P} 50|)$ & $\mathrm{C} 2$ \\
\hline ppp_eCor_env_NG_log(|P50|)_PC3 & $($ Env, trait $) \sim 1$ & $\log (|\mathrm{P} 50|)$ & $\mathrm{C}$ \\
\hline ppp_eCor_NG_log(Hv)_log $(|\mathrm{P} 50|)$ & (trait trait) $\sim 1$ & $\log (\mathrm{Hv})$ & og \\
\hline Spp_eCor_NG_log(Ks)_log(Hv) & (trait trait) $\sim 1$ & $\log (\mathrm{Ks})$ & g \\
\hline Spp_eCor_NG_log(Ks)_log $(|\mathrm{P} 50|)$ & $($ trait trait $) \sim 1$ & $\log (\mathbf{K s})$ & 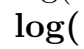 \\
\hline Spp_eCor_NG_log $(|\psi \min |) \_l o g(\mathrm{Hv})$ & (trait trait) ${ }^{\sim} 1$ & $\log (|\psi \min |)$ & ge \\
\hline 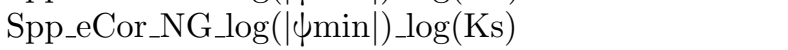 & (trait trait) $\sim 1$ & $\log (|\psi \min |)$ & \\
\hline Spp_eCor_NG_log $(|\psi \min |) \_l o g(|\mathrm{P} 50|)$ & (trait trait) ${ }^{\sim} 1$ & $\log (|\psi \min |)$ & \\
\hline Spp_eCor_GI_log(Hv)_log(|P50|)_PC1_PC2_PC3 & $($ trait trait $) \sim 1+$ group $*(\mathrm{PC} 1+\mathrm{PC} 2+\mathrm{PC} 3)$ & $\log (\mathrm{Hv})$ & \\
\hline Spp_eCor_GI_log(Ks)_log(Hv)_PC1_PC2_PC3 & $($ trait trait $) \sim 1+$ group $*(\mathrm{PC} 1+\mathrm{PC} 2+\mathrm{PC} 3)$ & $\log (\mathrm{Ks})$ & \\
\hline Spp_eCor__GI_log(Ks)_log(|P50|)_PC1_PC2_PC3 & (trait trait) $\sim 1+$ group $*(\mathrm{PC} 1+\mathrm{PC} 2+\mathrm{PC} 3)$ & $\log (\mathrm{Ks})$ & \\
\hline Spp_eCor_GI_log(| $|\mathrm{min}|)$ _log(Hv)_PC1_PC2_PC3 & $($ trait trait $) \sim 1+$ group $*(\mathrm{PC} 1+\mathrm{PC} 2+\mathrm{PC} 3)$ & $\log (|\psi \min |)$ & \\
\hline Spp_eCor_GI_log(|qmin|)_log(Ks)_PC1_PC2_PC3 & $($ trait trait $) \sim 1+$ group $*(\mathrm{PC} 1+\mathrm{PC} 2+\mathrm{PC} 3)$ & $\log (|\psi \min |)$ & \\
\hline Spp_eCor_GI_log(|భmin|)_log(|P50|)_PC1_PC2_PC3 & (trait trait) $\sim 1+$ group $*(\mathrm{PC} 1+\mathrm{PC} 2+\mathrm{PC} 3)$ & $\log (|\psi \min |)$ & \\
\hline
\end{tabular}

\section{Literature cited}

Jin, Y., \& Qian, H. (2019). V.PhyloMaker: an R package that can generate very large phylogenies for vascular plants. Ecography , 42(8), 1353-1359.

Paradis, E., \& Schliep, K. (2018). ape 5.0: an environment for modern phylogenetics and evolutionary analyses in R. Bioinformatics 35: 526-528.

Smith, S. A., \& Brown, J. W. (2018). Constructing a broadly inclusive seed plant phylogeny. Am. J. Bot. , 105(3), 302-314. 